\title{
Monstrous Moonshine from Orbifolds
}

\author{
Michael P. Tuite \\ Dublin Institute for Advanced Studies, 10 Burlington Road, Dublin 4, Ireland \\ Received July 17, 1990
}

\begin{abstract}
We consider the Monster Module of Frenkel, Lepowsky, and Meurman as a $Z_{2}$ orbifold of a bosonic string compactified by the Leech lattice. We show that the main Conway and Norton Monstrous Moonshine properties, stating that the Thompson series for each Monster group conjugacy class has a modular invariance group of genus zero, follow from an orbifold construction based on an orbifold group composed of Monster group elements. It is shown that a conjectured vacuum structure for the orbifold twisted sectors is sufficient to specify the modular group and the genus zero property for each Thompson series. It is also shown that the Power Map formula of Conway and Norton follows from the same vacuum structure. Finally, we demonstrate the validity of the vacuum conjectures for sectors twisted by Leech lattice automorphisms in many cases.
\end{abstract}

\section{Introduction}

One of the most intriguing examples of a conformal field theory (CFT) is the Monster Module described by Frenkel, Lepowsky, and Meurman [1]. They construct a meromorphic bosonic string theory on which the largest sporadic finite group $M$ known as the Fischer-Griess Monster [2] acts as a natural automorphism with the states at each Virasoro level forming a representation for $M$. In this paper we consider the famous Moonshine properties conjectured by Conway and Norton [3] which relate each conjugacy class of $M$ to a modular group whose fundamental region is always of genus zero. In a recent paper, Borcherds [4] has demonstrated that this genus zero property is correct using the theory of generalised Kac-Moody algebras [5]. In this paper we will show that these properties can also be understood in terms of orbifolds $[6,7]$ where the orbifold group is constructed from Monster group elements. We demonstrate that the vacuum structure of the corresponding twisted spaces is sufficient to ensure the genus zero property and the independent Power Map formula of Conway and Norton. 
We begin in Sect. 2 with a review of the Monster Module construction [1] as a bosonic string compactified by the Leech lattice which is then $Z_{2}$ orbifolded [8]. The resulting theory has no dimension 1 (massless) fields and the Monster group arises as the automorphism group of a closed algebra of dimension 2 fields. Section 2 also serves to illustrate some general features of orbifold constructions that we exploit later. In Sect. 3 we describe the main Conway and Norton Moonshine properties [3]. The section begins with a discussion on the meromorphic properties of a function invariant under a modular group with fundamental region of genus zero. For any such group, a unique invariant function with a single simple pole, known as a hauptmodul, always exists. The main Moonshine property is that for each $g \in M$ one can define a modular function $T_{g}$ (known as the Thompson series which depends only on the conjugacy class of $g$ ) which is a hauptmodul for some genus zero modular group. In Sect. 4 we interpret $T_{g}$ as an orbifold contribution and introduce the corresponding $g$ twisted sector. The actual method of construction of such a sector is not known but we can nevertheless describe some of the expected relevant properties on physical grounds. We discuss the origin of the modular invariance group for $T_{g}$ and show that the singularity structure of $T_{g}$ is determined by the vacuum properties of related twisted sectors. We introduce a set of vacuum conjectures which determine the full modular invariance group of $T_{g}$ and explain the genus zero Moonshine properties. In addition, we demonstrate that the vacuum conjectures imply the Power Map formula observed by Conway and Norton relating the invariance group of $T_{g}$ to that of $T_{g^{d}}$ for any power $d$. In Sect. 5 we discuss an important subgroup of $M$ related to Leech lattice automorphisms for which $T_{g}$ has a closed form. We discuss the validity of the vacuum conjectures of Sect. 4 for the strictly Leech lattice automorphism elements of this subgroup for which a standard twisted sector construction is possible. Section 6 concludes with a brief description of generalised Moonshine properties that we expect to follow also from the vacuum conjectures. We also briefly discuss the connection between the conjectured uniqueness of the Monster Module and the Moonshine properties. In the Appendix we give a description of a convenient set of inequivalent parabolic cusp points of the fundamental region for the basic modular group appearing in the Moonshine properties.

\section{The Monster Module}

In this section we review some features of the construction of the two-dimensional CFT associated with the Monster simple finite group known as the FLM Monster Module [1]. Most of the language adopted here follows the string theory exposition of the work of FLM in [8]. We will emphasise the orbifold aspects of this construction and highlight a few important general features that we will return to later.

The starting point is to define a Euclidean bosonic closed string compactified to a 24-dimensional torus $T^{24}$ [9]. The torus we choose is defined by quotienting $R^{24}$ with the Leech lattice $\Lambda$, the unique even self-dual lattice in 24 dimensions without vectors of length squared $2[10,11]$. The left-moving bosonic string variables $x^{i}(z)$ obey $x^{i}\left(e^{2 \pi i} z\right)=x^{i}(z)+2 \pi \beta$ with $z=e^{2 \pi i w}$, where $\operatorname{Re} w$ and $\operatorname{Im} w$ are "space" and "time" world-sheet coordinates and where $\beta \in \Lambda$. The mode expansion for $x^{i}(z)$ is

$$
x^{i}(z)=q^{i}-i p^{i} \ln z+i \sum_{n \neq 0} \frac{\alpha_{n}^{i}}{n} z^{-n}
$$


with

$$
\begin{aligned}
{\left[q^{i}, p^{j}\right] } & =i \delta^{i j}, \\
{\left[\alpha_{m}^{i}, \alpha_{n}^{j}\right] } & =m \delta^{i j} \delta_{m+n, 0} .
\end{aligned}
$$

A similar expression holds for $x^{i}(\bar{z})$ the right-moving part of the string. Since $\Lambda$ is even self-dual [11], the 1-loop partition function with world-sheet torus $w \sim w+1$ $\sim w+\tau$, takes the modular invariant form $Z(\tau) Z(\bar{\tau})$, where

$$
\begin{aligned}
Z(\tau) & =\operatorname{Tr}\left(q^{L_{0}}\right)=\frac{\Theta_{\Lambda}}{\eta^{24}}, \\
\Theta_{\Lambda} & =\sum_{\beta \in \Lambda} q^{\beta^{2} / 2}
\end{aligned}
$$

with $q=e^{2 \pi i \tau}$ and where $L_{0}$ is the normal ordered Virasoro Hamiltonian operator

$$
L_{0}=\frac{1}{2} p^{2}+\sum_{n=1}^{\infty} \alpha_{-n}^{i} \alpha_{n}^{i}-1
$$

The normal ordering constant $-C / 24$ is given by the tachyonic vacuum energy $24 \times \frac{1}{2} \sum n=-1$, where $C=24$ is the usual central charge. $\eta$ is the Dedekind eta function $\eta=q^{\frac{1}{24}} \prod_{n}\left(1-q^{n}\right)$ arising from the oscillator modes. $\Theta_{\Lambda}$ is the theta function associated with the Leech lattice $\Lambda$ and is a modular form of weight 12 $[11,12]$. The Hilbert space of states for this theory also factorises into meromorphic/antimeromorphic (in $z$ ) pieces. We may therefore consistently regard the left-moving string as a meromorphic CFT $[8,13]$ and ignore the rightmoving part from now on. The Hilbert space for this meromorphic CFT has the following Fock space representation

$$
H_{(+)}=\left\{\alpha_{-n_{1}}^{i_{1}} \ldots \alpha_{-n_{k}}^{i_{k}}|\beta\rangle\right\}, \quad n_{i} \in Z, \quad \beta \in \Lambda,
$$

where $|\beta\rangle$ is a highest weight state so that $\alpha_{n}|\beta\rangle=0$ for $n>0$ and $p^{i}|\beta\rangle=\beta^{i}|\beta\rangle$ [11]. The Fock space is graded by $L_{0}$ with integer level $\frac{1}{2} \beta^{2}+\sum n_{i}$. The subscript $(+)$ denotes the periodicity of states under $z \rightarrow e^{2 \pi i} z$ which implies for a typical state that

$$
e^{2 \pi i L_{0}}|\psi\rangle=|\psi\rangle
$$

since $L_{0}$ generates such a transformation. The integral grading of $L_{0}$ ensures that (2.6) is correct.

Returning to the partition function for these states we note that $Z(\tau)$ is a meromorphic and modular invariant function of $\tau . Z(\tau)$ may be found immediately in terms of the modular invariant function $j(\tau)$

$$
j(\tau)=\frac{E_{2}^{3}}{\eta^{24}}=\frac{1}{q}+744+196884 q+\ldots,
$$

where $E_{2}(\tau)$ is the Eisenstein modular form of weight 4 [12] corresponding to the $\Theta$ function for the $E_{8}$ root lattice. The $j$ function is the unique modular invariant function with a single simple pole at $q=0$ (up to a constant). This uniqueness property is discussed further in Sect. 3. It is a generalised version of this property for other modular groups that is the central issue in Monstrous Moonshine. 
Since $Z(\tau)$ also has a simple pole at $q=0$, due to the negative vacuum energy for $L_{0}$, we find that

$$
Z(\tau)=J(\tau)+24
$$

where we define $J(\tau)=j(\tau)-744$. The constant 24 reflects the existence of only 24 level one states $\left|\alpha_{-1}^{i}\right\rangle$ in this theory. Since the Leech lattice contains no length squared 2 vectors only these $U(1)$ bosonic states contribute.

The next stage of the Monster Module construction is the introduction of a $Z_{2}$ twist operation $g: X^{i} \rightarrow-X^{i}$ which acts as an automorphism on the states (2.5) of the theory [1]. We can construct a new CFT corresponding to string propagation on the quotient of $T^{24}$ by $g$. Geometrically, the manifold $T^{24}$ becomes an orbifold $T^{24} / Z_{2}$ which is a manifold except at the conical singularities due to any fixed points of $g[6,7]$. In this case, these are labelled by $\Lambda / 2 \Lambda$ and have multiplicity $2^{24}$. The states of this new orbifold or "twisted" theory are composed partly of the $g$ invariant projection of the original Hilbert space $H_{(+)}$and new twisted states which must be introduced for modular consistency.

The original Hilbert space $H_{(+)}$can be decomposed into \pm 1 eigenstates of $g$ as follows

$$
\begin{aligned}
& H_{(+)}^{+}=\left\{\alpha_{-n_{1}}^{i_{1}} \ldots \alpha_{-n_{2 k}}^{i_{2 k}}(|\beta\rangle+|-\beta\rangle)\right\} \cup\left\{\alpha_{-n_{1}}^{i_{1}} \ldots \alpha_{-n_{2 k+1}}^{i_{2 k+1}}(|\beta\rangle-|-\beta\rangle)\right\}, \\
& H_{(+)}^{-}=\left\{\alpha_{-n_{1}}^{i_{1}} \ldots \alpha_{-n_{2 k}}^{i_{2 k}}(|\beta\rangle-|-\beta\rangle)\right\} \cup\left\{\alpha_{-n_{1}}^{i_{1}} \ldots \alpha_{-n_{2 k+1}}^{i_{2 k+1}}(|\beta\rangle+|-\beta\rangle)\right\},
\end{aligned}
$$

where defining the projection operator $\mathscr{P}=\frac{1}{2}(1+g)$ we have $H_{(+)}^{+}=\mathscr{P} H_{(+)}$.

Consider now the partition function for the $g$ invariant states $H_{(+)}^{+}$

$$
\operatorname{Tr}_{H(+)}\left(\mathscr{P} q^{L_{0}}\right)=\frac{1}{2} \operatorname{Tr}_{\boldsymbol{H ( + )}}\left(q^{L_{0}}\right)+\frac{1}{2} \operatorname{Tr}_{\boldsymbol{H}(+)}\left(g q^{L_{0}}\right) .
$$

The first trace is the original partition function $Z(\tau)$ of $(2.3)$. The second can be interpreted as a closed string contribution periodic in the "space" direction but twisted by $g$ in the "time" direction. We denote this contribution in the standard way by $-\square$. Calculating this trace from $(2.9 \mathrm{~b})$ we have [8]

$$
-\square=q^{-1} \prod_{n}\left(1+q^{n}\right)^{-24}=2^{12}\left[\frac{\eta}{\Theta_{2}}\right]^{12} \text {. }
$$

Similarly, we can denote the original trace of $(2.3)$ by $+\square$. Performing the modular transformation $S: \tau \rightarrow-1 / \tau$ on $-\square$ the space and time boundary conditions are interchanged to give a contribution $+\square$ corresponding to states twisted by $g$ in the space direction but periodic in the time direction. Such a contribution arises from a new twisted sector with Hilbert space $H_{(-)}$which is introduced below. In general, we can consider a Hilbert space $H_{h}$ of states twisted by some group element $h$. Then performing the trace $\operatorname{Tr}_{H_{h}}\left(g q^{L_{0}}\right)=g \square$, with some appropriate $L_{0}$, the boundary conditions transform under a general modular transformation $\tau \rightarrow(a \tau+b) /(c \tau+d)$ to give $g^{d} h^{-b}{ }_{g^{-c} h^{a}}$ for $h, g$ commuting (up to
phase factors) $[6,14]$.

To define the twisted Hilbert space we consider a closed string field $x^{i}(z)$ on $T^{24} / Z_{2}$ with boundary conditions $x^{i}\left(e^{2 \pi i} z\right)=-x^{i}(z)+\beta^{i}, \beta^{i} \in \Lambda$. The mode expan- 
sion is now

$$
x^{i}(z)=\tilde{q}^{i}+i \sum_{n \in Z+\frac{1}{2}} \frac{\alpha_{n}^{i}}{n} z^{-n},
$$

where the oscillator modes obey the same commutation relations given in (2.1). There is a similar expansion for $x^{i}(\bar{z})$ involving the same centre of mass coordinates $\tilde{q}^{i}$ which take values in the $2^{24}$-dimensional fixed point space $\Lambda / 2 \Lambda$ of $g$. Each fixed point corresponds to one Fock vacuum $\left|\tilde{q}_{a}\right\rangle\left(a=1 \ldots 2^{24}\right)$, where

$$
\tilde{q}\left|\tilde{q}_{a}\right\rangle=\tilde{q}_{a}\left|\tilde{q}_{a}\right\rangle, \quad \tilde{q}_{a} \in \Lambda / 2 \Lambda .
$$

Twisted states are then constructed by acting on these vacua with left and right creation operators. Since we are interested in meromorphic twisted states only $2^{12}$ of these Fock vacua are actually employed. These vacua are denoted by $\left|\sigma_{a}\right\rangle$, $a=1 \ldots 2^{12}$, and form a $2^{12}$-dimensional representation space $T$ of a $Z_{2}$ central extension of $\Lambda / 2 \Lambda$. This in turn is induced by a $Z_{2}$ central extension of $\Lambda$ with a two-cycle $\varepsilon(\alpha, \beta)$ for $\alpha, \beta \in \Lambda$ familiar from the vertex construction for the states $|\beta\rangle$ of (2.5). Some of the details of this construction are discussed in Sect. 5.

The twisted Hilbert space $H_{(-)}$is now defined by the Fock space representation

$$
H_{(-)}=\left\{\alpha_{-n_{1}}^{i_{1}} \ldots \alpha_{-n_{k}}^{i_{k}}|0\rangle \otimes\left|\sigma_{a}\right\rangle\right\}, \quad n_{i} \in Z+\frac{1}{2}, \quad a=1 \ldots 2^{12},
$$

where $\alpha_{n}^{i}|0\rangle=0, n>0$. These states are graded by the Virasoro Hamiltonian

$$
L_{0}=\sum_{n=\frac{1}{2}}^{\infty} \alpha_{-n}^{i} \alpha_{n}^{i}+\frac{1}{2}
$$

with half integer level $\frac{1}{2}+\sum n_{i}$. The appropriate normal ordering constant for $L_{0}$ can be found by zeta function regularisation to be $24 \times \frac{1}{2} \sum\left(n+\frac{1}{2}\right)=\frac{1}{2}$. (In general we have $\sum(n+r)=\frac{1}{12}(-1+6 r(1-r))$ [7].) The automorphism $g$ also induces a group operation on $H_{(-)}$which can be decomposed into \pm 1 eigenstates as before

$$
\begin{aligned}
& H_{(-)}^{+}=\left\{\alpha_{-n_{1}}^{i_{1}} \ldots \alpha_{-n_{2 k+1}}^{i_{2 k+1}}|0\rangle \otimes\left|\sigma_{a}\right\rangle\right\}, \\
& H_{(-)}^{-}=\left\{\alpha_{-n_{1}}^{i_{1}} \ldots \alpha_{-n_{2 k}}^{i_{2 k}}|0\rangle \otimes\left|\sigma_{a}\right\rangle\right\},
\end{aligned}
$$

where $\mathscr{P} H_{(-)}=H_{(-)}^{+}$and the twisting of a typical state of $H_{(-)}$is expressed by

$$
g e^{2 \pi i L_{0}}|\psi\rangle=|\psi\rangle,
$$

which is easily verified by noting that the states of $H_{(-)}^{+}\left(H_{(-)}^{-}\right)$have levels in $Z\left(Z+\frac{1}{2}\right)$.

We may now compute the partition function for $H_{(-)}^{+}$to be

where we find [8]

$$
\operatorname{Tr}_{H_{(-)}^{+}}\left(q^{L_{0}}\right)=\frac{1}{2}(+\square+-\underline{\square}),
$$

$$
\begin{aligned}
+\underline{\square} & =\operatorname{Tr}_{H_{(-)}}\left(q^{L_{0}}\right) \\
& =2^{12} q^{1 / 2} \prod_{n}^{\infty}\left(1-q^{n-\frac{1}{2}}\right)^{-24}=2^{12}\left[\frac{\eta}{\Theta_{4}}\right]^{12}, \\
-\square & =\operatorname{Tr}_{H_{(-)}}\left(g q^{L_{0}}\right) \\
& =-2^{12} q^{1 / 2} \prod_{n}^{\infty}\left(1+q^{n-\frac{1}{2}}\right)^{-24}=-2^{12}\left[\frac{\eta}{\Theta_{3}}\right]^{12},
\end{aligned}
$$


where the vacuum degeneracy contributes $2^{12}$. The boundary conditions associated with these traces (2.11), (2.19) transform amongst themselves under modular transformation. Therefore, the full partition function is the modular invariant

$$
\begin{aligned}
Z_{t w}(\tau)= & \frac{1}{2}(+\square+-\square) \\
& +\frac{1}{2}(+\square+-\square)
\end{aligned}
$$

which gives $Z_{t w}(\tau)=J(\tau)$ on substitution.

In general, to ensure that (2.20) is correct, we must check the consistency of any phases that may arise under modular transformations. Specifically, we must ensure that a modular transformation which fixes boundary conditions leaves the appropriate trace invariant [14]. Thus $+\square$ (and $-\square$ ) must be invariant under $T^{2}: \tau \rightarrow \tau+2$. We find therefore that the stability group of fixing transformations for $-\square$ is generated by $T$ and $S T^{2} S$ [which generate the modular group $\Gamma_{0}(2)$ ]. The necessary existence of such a stability group for modular consistency is a general feature of orbifold constructions. In general, this leads to constraints on the automorphisms that can be employed to form a modular consistent theory [14, 15]. In Sect. 4 we will return to this feature of orbifold constructions. In the present case it is easy to check that these conditions are satisfied, i.e. the partition functions $+\square$ and $-\square$ are $T^{2}$ invariant since the twisted sector levels are half integrally graded.

The orbifold theory now constructed is the Monster Module of FLM [1]. The states of the theory are given by the $g$ invariant Hilbert space $H_{(+)}^{+} \oplus H_{(-)}^{+}$denoted as $V^{\natural}$ by FLM. It is the states of this theory on which the Monster group $M$ has its action.

One of the motivations of FLM for looking at such a structure was the observation by McKay and Thompson [16] that the coefficients of the modular invariant function $j(\tau)$ are sums of dimensions of representations of $M$. Thus the coefficient of $q^{1}$ in (2.7) is the sum of the trivial and lowest dimensional representations $196884=1+196883$. Likewise, all higher coefficients of $j(\tau)$ can be expanded as sums of dimensions of $M$ representations [16]. The only exception is the constant term 744 of $j$. By construction the Monster Module has no states of level 1 and so this exceptional case is excluded. The Monster group $M$ now arises naturally out of the commutator algebra of the 196884 level 2 operators which includes the Energy-Momentum operator $T(z)=\frac{1}{2} \partial x \cdot \partial x$ [8]. Taking Fourier components of these level 2 operators, denoted $\phi_{n}^{i}$, a symmetric non-associative cross-bracket operation can be defined as follows:

$$
\phi_{m}^{i} \times \phi_{n}^{j}=\frac{1}{2}\left[\phi_{m+1}^{i}, \phi_{n-1}^{j}\right]+\frac{1}{2}\left[\phi_{n+1}^{j}, \phi_{m-1}^{i}\right] .
$$

The purpose of this cross-bracket is to remove any level 3 operators which necessarily arise in the commutation of two level 2 operators. Thus the crossbracket algebra for level 2 operators closes to form the following algebra $[1,8]$ :

$$
\begin{aligned}
& \phi_{m}^{i} \times \phi_{n}^{j}=b^{i j k} \phi_{m+n}^{k}+\frac{1}{6} \delta^{i j} L_{m+n}+\frac{m^{2}}{2} \delta^{i j} \delta_{m+n, 0}, \\
& L_{n} \times \phi_{m}^{i}=2 \phi_{m+n}^{i}, \\
& L_{m} \times L_{n}=2 L_{m+n}+6 m^{2} \delta_{m+n, 0},
\end{aligned}
$$


where $b^{i j k}$ are some coefficients symmetric in $i, j$ and $L_{n}$ is the Virasoro generator $\frac{1}{2} \sum \alpha_{-n} \alpha_{n+m}$. Equations (2.22b) and (2.22c) are symmetrized versions of the usual level 2 conformal properties of $\phi_{n}^{i}$ and the Virasoro algebra.

The closed algebra (2.22a) is an affine version of the Griess algebra [2] which can be recovered by setting $m=n=0$. The automorphism group of this algebra was shown by Griess to be the largest finite simple sporadic group known as the Fischer-Griess Monster group $M$ [2]. The level 2 operators $\left\{\phi_{n}^{i}\right\}$ form the lowest dimensional 196883 adjoint representation of $M$. The remaining level 2 operator, $L_{0}$, is a singlet under $M$. Therefore, the states of the theory form representations for $M$ at each level given by $L_{0}$. This is the basis for the original observations of McKay and Thompson about the coefficients of $j(\tau)$.

For much of the discussion in the following two sections, the explicit form of the elements of $M$ will not be required. What is important, for our immediate purposes, is that the states at each level form representations of $M$. We can, however, identify here one important element: the involution (order two element) $i$ under which all untwisted states have eigenvalue +1 and twisted states have eigenvalue -1 . $i$ can be thought of as a "fermion number" operator. The correct identification of the centralizer $C$ of $i$ in $M$ (all elements of $M$ that commute with $i$ ) forms a crucial part of the FLM construction. The monster group is characterized by the fact that $C$ together with another involution $\sigma$ generate $M[2,1]$. In Sect. 5 we will consider $C$ in more detail.

\section{Hauptmoduls and Monstrous Moonshine}

We will now describe the famous Moonshine properties of Conway and Norton [3] in terms of the Monster Module $V^{\natural}$ of the last section. In order to do this we will return initially to the $j$ modular function of (2.6). We will prove the uniqueness property of $j$ in sufficiently general terms to be of use in both stating and understanding Monstrous Moonshine.

The $j$ modular function is a meromorphic function of $\tau$ invariant under all modular transformations $\tau \rightarrow(a \tau+b) /(c \tau+d), a d-b c=1$ for $a, b, c, d \in Z$, generated by the full modular group $\Gamma=P S L(2, Z)=S L(2, Z) /\{ \pm 1\}$. The $\tau$ variable is defined over the upper half plane $H$ which is in general invariant under the larger class of Möbius transformations generated by $\operatorname{PSL}(2, R)$ of which $\Gamma$ is a discrete subgroup. We may therefore factor $H$ by $\Gamma$ to obtain the usual fundamental region $\mathscr{F}=H / \Gamma$ conventionally taken as $|\tau| \geqq 1,|\operatorname{Im} \tau| \leqq \frac{1}{2}$ as shown in Fig. 1. Adding in the parabolic cusp point at $i \infty(q=0)$ and identifying edges under $\Gamma$ we obtain the compactified fundamental region $\overline{\mathscr{F}}$. This is clearly the Riemann sphere of genus zero. The modular invariance of $j(\tau)$ under $\Gamma$ means that $j$ is single-valued on $\overline{\mathscr{F}}$ (including the elliptic fixed points $\varrho=e^{i \pi / 3}=S T \varrho$ and $i=S i$ [17]). Therefore, $j$ provides a map from $\overline{\mathscr{F}}$ to $C \cup\{i \infty\}$. In fact, this map is an isomorphism and explicitly realizes the spherical topology of $\overline{\mathscr{F}}$. To see this, we will introduce a standard general theorem which will be exploited greatly later on.

Consider a more general situation as follows. Let $\Gamma_{1}$ be some subgroup of $\operatorname{PSL}(2, R)$ with fundamental region $\mathscr{F}_{1}$. We will assume that $\Gamma_{1}$ is commensurable with $\Gamma$, i.e. the index of $\Gamma \cup \Gamma_{1}$ in $\Gamma$ and $\Gamma_{1}$ is finite [the dimensions of $\Gamma /\left(\Gamma \cup \Gamma_{1}\right)$ and $\Gamma_{1} /\left(\Gamma \cup \Gamma_{1}\right)$ are finite $]$. This implies that $\Gamma_{1}$ is also discrete like $\Gamma$. We may compactify $\mathscr{F}_{1}$ by adding in any parabolic cusp points and identifying edges under $\Gamma_{1}$ to obtain a compact Riemann surface $\overline{\mathscr{F}}_{1}$ of some genus [17]. (A parabolic cusp point is any 


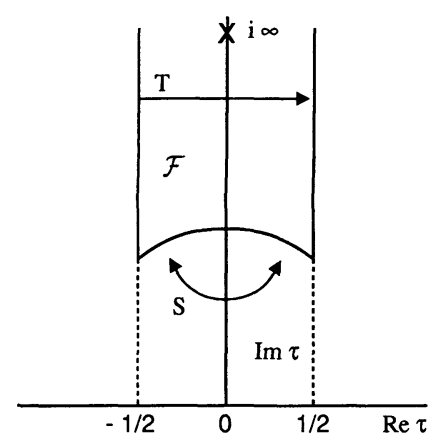

Fig. 1. The fundamental region $\mathscr{F}$ for the full modular group $\Gamma$ is equivalent to the Riemann sphere on identifying edges and adding in the point at infinity

point of $R \cup\{i \infty\}$ which is fixed by an element of $\Gamma_{1}$.) Consider next some meromorphic $\Gamma_{1}$ invariant function $f(\tau)$. This induces a meromorphic function $\bar{f}(\tau)$ on $\overline{\mathscr{F}}_{1}$. For a regular point $\tau$ of $\mathscr{F}_{1}$ this is just $f(\tau)$ whereas for elliptic and parabolic fixed points a little more care is required to define a local coordinate patch on $\overline{\mathscr{F}}_{1}$ (see [17] for details). We can now state the following general theorem:

Hauptmodul Theorem. Let $j_{1}(\tau)$ be a meromorphic $\Gamma_{1}$ invariant function with induced function $\bar{j}_{1}(\tau)$ on $\overline{\mathscr{F}}_{1}$. Then $\bar{j}_{1}(\tau)$ has a unique simple pole on $\overline{\mathscr{F}}_{1}$ if and only if $\bar{j}_{1}: \overline{\mathscr{F}}_{1} \rightarrow C \cup\{i \infty\}$ is an isomorphism, i.e. $\overline{\mathscr{F}}_{1}$ is the Riemann sphere of genus zero.

The proof of this theorem relies on the property that the number of zeros equals the number of poles for a meromorphic function on a compact Riemann surface. Suppose first that $\bar{j}_{1}(\tau)$ has a unique simple pole, $\tau=\tau_{\infty}$ on $\overline{\mathscr{F}}_{1}$. Then choosing a contour $\gamma$ about the pole but sufficiently small to exclude any zeros we find by Cauchy's theorem that

$$
\frac{1}{2 \pi i} \oint_{\gamma} \frac{d \overline{j_{1}}}{\overline{j_{1}}}=-1
$$

Since $\overline{\mathscr{F}}{ }_{1}$ is compact, we may also evaluate (3.1) as a sum of residues due to the poles and zeros of $\bar{j}_{1}(\tau)$ outside of $\gamma$. Therefore, $\bar{j}_{1}(\tau)$ has a unique zero of order 1 on $\overline{\mathscr{F}}_{1}$.

Consider next any $c \in C$. Then $\overline{f_{c}}=\overline{j_{1}}-c$ is $\Gamma_{1}$ invariant with a unique simple pole at $\tau_{\infty}$ and therefore also vanishes at some unique point $\tau_{c}$, i.e. $\bar{j}_{1}\left(\tau_{c}\right)=c$. Finally, $\bar{j}_{1}(\tau)$ is infinite at one point $\tau_{\infty}$ of $\overline{\mathscr{F}}_{1}$. Therefore, $\bar{j}_{1}: \overline{\mathscr{F}}_{1} \rightarrow C \cup\{i \infty\}$ is an isomorphism and hence $\overline{\mathscr{F}}_{1}$ is the Riemann sphere.

Likewise, if $\bar{j}_{1}(\tau)$ is an isomorphism then it vanishes at only one point $\tau_{0}$ of $\overline{\mathscr{F}}_{1}$. If the zero is of order $n$ (implying that the unique pole is of order $n$ also) then expanding we find

$$
\bar{j}_{1}(\tau)=\left(\tau-\tau_{0}\right)^{n}\left(a_{n}+a_{n+1}\left(\tau-\tau_{0}\right)+\ldots\right)
$$

for $\left|\tau-\tau_{0}\right|$ sufficiently small. It is easy to see that $\bar{j}_{1}(\tau)$ cannot be single valued near $\tau_{0}$ if $n>1$. This follows by noticing that a closed contour about $\tau_{0}$ in $\overline{\mathscr{F}}_{1}$ encircles the origin of the complex plane $n$ times and therefore must self-intersect at least $n-1$ times implying multi-valuedness. Therefore, $n=1$ and $\bar{j}_{1}(\tau)$ has a unique pole of order 1 . 
The power of this theorem is that we may express any meromorphic $\Gamma_{1}$ invariant function $f(\tau)$ as a rational function of $j_{1}(\tau)$ :

$$
f(\tau)=\frac{\sum_{l}^{m} a_{l} j_{1}^{l}}{\sum_{k}^{n} b_{k} j_{1}^{k}},
$$

where the coefficients $a_{l}, b_{k}$ are chosen such that the residues for the zeros and poles of $f(\tau)$ are matched. The set of all such $\Gamma_{1}$ invariant meromorphic functions is called a genus zero function field and $\Gamma_{1}$ is called a genus zero modular group. The special function $j_{1}(\tau)$ is called a hauptmodul or a generator for this genus zero function field.

As an example of the property (3.3) one can show that any two hauptmoduls $j_{1}, j_{1}^{\prime}$ for a genus zero group $\Gamma_{1}$ must be related by

$$
j_{1}^{\prime}(\tau)=\frac{A j_{1}+B}{C j_{1}+D}
$$

for some complex numbers $A, B, C, D$ chosen to give the correct pole and zero for $j_{1}^{\prime}(\tau)$. This relation is a $\operatorname{PSL}(2, C)$ transformation on $j_{1}$ demonstrating the well known result that the automorphism group of the Riemann sphere is $\operatorname{PSL}(2, C)$.

Returning to the case of the $j$ function for $\Gamma$, the full modular group, we can see from Eq. (2.6) that $j$ has a unique simple pole at $q=0$. This corresponds to a simple pole on $\mathscr{F}$ since the invariance of $j$ under $T$ ensures that for $\operatorname{Re} \tau=$ const in $\mathscr{F}$, one circuit of the contour $|q|=$ const is executed on $\overline{\mathscr{F}}$. Therefore, the hauptmodul $j(\tau)$ is the unique $\Gamma$ invariant function with a pole at $q=0$ as earlier stated. To emphasis the importance of these ideas for $\Gamma$ we can consider the example of the modular invariant function $f(\tau)=\left(\theta_{2}^{4}-\theta_{3}^{4}+\theta_{4}^{4}\right)^{6} / \eta^{24}$. This has no poles and vanishes at $q=0$ on $\bar{F}$ and therefore must vanish everywhere giving the Jacobi triple identity. Likewise, the fact that all $\Gamma$ modular forms can be expressed as polynomials in the basic Eisenstein series $E_{2}$ and $E_{3}$ follows from the uniqueness of $j(\tau)$ [12].

Let us return now to the $Z_{2}$ orbifold Monster Module $V^{\natural}$ of Sect. 2 and define the Thompson series for an element $g$ of $M$. The states of $V^{\natural}$ transform under (reducible) representations of $M$. Therefore, we may define the following trace:

$$
T_{g}(\tau)=\operatorname{Tr}_{V^{\natural}}\left(g q^{L_{0}}\right)
$$

which is known as the Thompson series for $g \in M$ [16]. As an example, we can calculate immediately the series for the involution $i$ defined in Sect. 2,

$$
T_{i}(\tau)=\operatorname{Tr}_{\left.H_{\left(^{+}\right.}^{+}\right)}\left(q^{L_{0}}\right)-\operatorname{Tr}_{H_{\left(^{+}\right.}^{+}}\left(q^{L_{0}}\right),
$$

which on substituting (2.8), (2.12), and (2.19) gives

$$
T_{i}(\tau)=2^{12}\left[\frac{\eta}{\Theta_{2}}\right]^{12}+24=\left[\frac{\eta(\tau)}{\eta(2 \tau)}\right]^{24}+24
$$

In general, expanding in $q$ we find

$$
T_{g}(\tau)=\frac{1}{q}+0+\left(1+\chi_{1}(g)\right) q+\ldots,
$$

where $\chi_{1}(g)$ is the character for $g$ in the 196883 representation. Likewise, the higher coefficients are sums of characters of $g$ in the various representations occurring at 
each level. For each of the 172 conjugacy classes of $M$ there is such a Thompson series. Conway and Norton [3] calculated the coefficients for each of these series up to $q^{10}$ and formulated their Moonshine properties as a result. They conjectured that each Thompson series is a hauptmodul for a genus zero modular group $\Gamma_{g}$ commensurable with $\Gamma$, i.e. each $T_{g}(\tau)$ is invariant under some fixing modular group $\Gamma_{g}$ and that $\overline{\mathscr{F}}_{g}=\bar{H} / \Gamma_{g}$ is of genus zero where $T_{g}$ is the hauptmodul with a simple pole at $q=0$. [All the states of $V^{\natural}$ are of integer level so that $T_{g}$ is $T$ invariant. Therefore, as for the $\Gamma$ invariant function $j(\tau)$, the pole at $q=0$ is of order 1 on $\overline{\mathscr{F}}_{g}$.] The uniqueness of the hauptmodul allowed Conway and Norton to list all the modular functions $T_{g}(\tau)$ and to find many non-trivial formulae amongst these functions.

To describe the $\Gamma_{g}$ groups relevant to the Moonshine properties we must first define a number of modular groups of interest.

$\Gamma_{0}(N)$ : The group of matrices $\gamma \in \Gamma$ of the form

$$
\gamma=\left(\begin{array}{cc}
a & b \\
c N & d
\end{array}\right), \quad \operatorname{det} \gamma=1,
$$

where $a, b, c, d \in Z$.

The full structure of the Normalizer group of $\Gamma_{0}(N)$ in $\operatorname{PSL}(2, R), \mathscr{N}(N)$ $=\left\{\varrho \in P S L(2, R) \mid \varrho \Gamma_{0}(N) \varrho^{-1}=\Gamma_{0}(N)\right\}$, is also required to describe $\Gamma_{g}$. Here we will quote from the elegant description of $\mathscr{N}(N)$ given by Conway and Norton [3].

Let $h \in Z$ where $h^{2} \mid N\left(h^{2}\right.$ divides $\left.N\right)$ and let $N=n h$. Then we define the following sets of matrices.

$\Gamma_{0}(n \mid h)$ : The group of matrices of the form

$$
\left(\begin{array}{cc}
a & \frac{b}{h} \\
c n & d
\end{array}\right), \quad \operatorname{det}=1,
$$

where $a, b, c, d \in Z$. For $h$ the largest divisor of 24 for which $h^{2} \mid N, \Gamma_{0}(n \mid h)$ forms a subgroup of $\mathscr{N}(N)$. The group is generated by the cosets $C_{1}=\left\{\gamma \in \Gamma_{0}(n \mid h) \mid a=b=d\right.$ $=1 \bmod h, c=0 \bmod h\}$ and $C_{2}=\left\{\gamma \in \Gamma_{0}(n \mid h) \mid a=c=d=1 \bmod h, b=0 \bmod h\right\}$ [3]. Note that each element of $C_{1}$ (or $C_{2}$ ) can be expressed as $T^{\frac{1}{h}}: \tau \rightarrow \tau+\frac{1}{h}$ (or respectively $\left.S T^{n} S\right)$ times an element of $\Gamma_{0}(N)$.

$W_{e}$ : The set of matrices for a given integer $e$

$$
\left(\begin{array}{cc}
a e & b \\
c N & d e
\end{array}\right), \quad \operatorname{det}=e, \quad e \| N,
$$

where $a, b, c, d \in Z . e \| N$ denotes the property that $e \mid N$ and the greatest common divisor $\left(e, \frac{N}{e}\right)=1$. The set $W_{e}$ forms a single coset of $\Gamma_{0}(N)$ in $\mathscr{N}(N)$ with $W_{1}=\Gamma_{0}(N)$. It is straightforward to show that (up to scale factors)

$$
\begin{aligned}
W_{e}^{2} & =1 \bmod \left(\Gamma_{0}(N)\right), \\
W_{e_{1}} W_{e_{2}} & =W_{e_{2}} W_{e_{1}}=W_{e_{3}} \bmod \left(\Gamma_{0}(N)\right), \quad e_{3}=\frac{e_{1} e_{2}}{\left(e_{1}, e_{2}\right)^{2}} .
\end{aligned}
$$


The coset $W_{e}$ is referred to as an Atkin-Lehner (AL) involution for $\Gamma_{0}(N)$. The simplest example is the Fricke involution $W_{N}$ with coset representative $\left(\begin{array}{cc}0 & 1 \\ -N & 0\end{array}\right)$ which generates $\tau \rightarrow-1 / N \tau$. This transformation is already familiar in another string theory context $[18,19]$.

$w_{e}$ : The set of matrices for a given integer $e$ of the form

$$
\left(\begin{array}{cc}
a e & \frac{b}{h} \\
c n & d e
\end{array}\right), \quad \operatorname{det}=e, \quad e \| \frac{n}{h},
$$

where $a, b, c, d \in Z$. The set $w_{e}$ is called an Atkin-Lehner (AL) involution for $\Gamma_{0}(n \mid h)$. The properties (3.12) are similarly obeyed by $w_{e}$ with $\Gamma_{0}(N)$ replaced by $\Gamma_{0}(n \mid h)$.

$\mathscr{N}(N)$ : The Normalizer of $\Gamma_{0}(N)$ in $\operatorname{PSL}(2, R)$ is generated by adjoining to $\Gamma_{0}(n \mid h)$ all its AL involutions $w_{e_{1}}, w_{e_{2}}, \ldots$, where $h$ is the largest divisor of 24 and $h^{2} \mid N, N=n h$.

$\Gamma_{0}(n \mid h)+e_{1}, e_{2}, \ldots$ : This denotes the group obtained by adjoining to $\Gamma_{0}(n \mid h) a$ particular subset of AL involutions $w_{e_{1}}, w_{e_{2}}, \ldots$. Clearly, this forms a subgroup of $\mathcal{N}(N)$.

All of the groups defined above are commensurable with the full modular group $\Gamma$. We are now in a position to state the main Moonshine properties of Conway and Norton [3, 20].

Moonshine Properties. Let $g \in M$ of order $n$.

(a) The Thompson series $T_{g}(\tau)$ is invariant up to a phase of order $h$ under a subgroup of $\mathscr{N}(N)$ of the form $\Gamma_{0}(n \mid h)+e_{1}, e_{2}, \ldots$, where $h|24, h| n$, and $N=n h$.

(b) The subgroup $\Gamma_{g}$ of these transformations which fixes $T_{g}$ (and contains $\Gamma_{0}(N)$ ) is of genus zero where $T_{g}$ is the hauptmodul with a simple pole at $q=0$.

Let us examine how these properties apply to an element of prime order $p$. Part (a) tells us that $h=1$ (excepting only one class of $M$ with $n=3$ and $h=3$ [3] which we exclude from the following remarks). Part (b) tells us that the fixing group $\Gamma_{g}$ contains $\Gamma_{0}(p)$ and is contained in $\mathscr{N}(p)$. However, $\mathscr{N}(p)$ contains only one nontrivial element, the Fricke involution $W_{p}: \tau \rightarrow-1 / p \tau . \mathscr{N}(p)$ is therefore found by adjoining $W_{p}$ to $\Gamma_{0}(p)$ which is denoted by $\Gamma_{0}(p)+$. Therefore, we find that either $\Gamma_{g}=\Gamma_{0}(p)$ or $\Gamma_{0}(p)+$. It is well known that $\Gamma_{0}(p)$ is of genus zero only when $(p-1) \mid 24$ for $p$ prime [21]. The corresponding hauptmodul is just

$$
\left[\frac{\eta(\tau)}{\eta(p \tau)}\right]^{r}+r
$$

where $(p-1) r=24$. Invariance under $\Gamma_{0}(p)$ is ensured for $r$ an integer. The constant in (3.14) is chosen so that the $q^{0}$ coefficient is zero. For each $p$, with $(p-1) \mid 24$ there is indeed a conjugacy class of $M$, labelled $p-$, with the Thompson series (3.14). Recalling our earlier example with the involution element $i$ we obtain (3.7) from (3.14) for $r=24$ and $p=2$. In Sect. 5 we shall explicitly reproduce the remaining Thompson series of this form from Leech lattice automorphisms.

The primes for which $\Gamma_{0}(p)+$ is of genus zero have been found by Ogg [22] to be precisely the primes that divide the order of $M$. These are all primes $p$ with $2 \leqq p \leqq 31$ and $p=41,47,59,71$. For each of these primes there is a conjugacy class, labelled $p+$, whose Thompson series is the hauptmodul for this group. A subset of these classes with $(p+1) \mid 24$ associated with Leech lattice automorphisms will be explicitly calculated in Sect. 5 . 
In general, there are 174 possible discrete subgroups of genus zero which contain some $\Gamma_{0}(N)$ and are contained in $\Gamma_{0}(N)+e_{1}, e_{2}, \ldots$ [3]. There are 171 classes for $M$, each of which has a unique Thompson series corresponding to one of these discrete groups (except for two classes of order 27 which share the same Thompson series). The remaining 3 genus zero groups $(N=25,49,50)$ correspond to the socalled "ghost elements" of $M$ [3].

There are many remarkable relationships found by Conway and Norton between different Thompson series many of which owe their origin to the hauptmodul property (3.2). In particular, the so-called replication formulae reveal that all coefficients of any Thompson series $T_{g}$ can be expressed in terms of the first five of $T_{g}, T_{g^{2}}, T_{g^{4}}, \ldots$ by recursion formulae. Borcherds has recently shown that these properties can also be deduced from determinant formulae based on generalised Kac-Moody algebras and hence the hauptmodul properties follow [4].

Another interesting and important consequence of the hauptmodul property is the behaviour of $T_{g}$ under a transformation generated by an element $\varrho$ of the normalizer of $\Gamma_{g}$ in $P S L(2, R)$. The modular function $T_{g}^{Q}=T_{g}(\varrho(\tau))$ is meromorphic on $H$ with a simple pole at $\varrho^{-1}(i \infty)$. In addition, $T_{g}^{e}$ is $\Gamma_{g}$ invariant since $\varrho \Gamma_{g} \varrho^{-1}=\Gamma_{g}$. Therefore, by the Hauptmodul Theorem, $T_{g}$ is also a hauptmodul for $\Gamma_{g}$. By (3.4) we know that

$$
T_{g}^{e}(\tau)=\frac{A T_{g}+B}{C T_{g}+D}
$$

for some complex numbers $A, B, C, D$. As an example of this, consider the class $p-$ with $(p-1) \mid 24$, which has Thompson series (3.14). The only non-trivial element of $\mathcal{N}(p)$ is $W_{p}$. Defining $\phi_{g}=T_{g}-r$ we find that $\phi_{g}$ is inverted by $W_{p}$ :

$$
W_{p}\left(\phi_{g}(\tau)\right)=\left[\frac{\eta(-1 / p \tau)}{\eta(-1 / \tau)}\right]^{r}=\frac{p^{\frac{r}{2}}}{\phi_{g}(\tau)} .
$$

In general, the normalizer of $\Gamma_{g}$ in $\operatorname{PSL}(2, R)$ contains the Fricke involution $W_{N}$. Because $W_{N}^{2}=1 \bmod \left(\Gamma_{0}(N)\right)$ we find from (3.15) that either $T_{g}$ is $W_{N}$ invariant (i.e. $W_{N} \in \Gamma_{g}$ ) or is inverted according to

$$
W_{N}\left(\phi_{g}(\tau)\right)=\frac{c}{\phi_{g}(\tau)}, \quad W_{N} \notin \Gamma_{g},
$$

where $\phi_{g}(\tau)=T_{g}(\tau)-T_{g}(0)$ and $c \in C$.

Equations (3.16) and (3.17) allow us to determine the phase referred to in part (a) of the Moonshine properties. Assuming that $T_{g}(\tau)$ is $\Gamma_{0}(N)$ invariant, it follows from our remarks about the cosets $C_{1}$ and $C_{2}$ that it is sufficient to describe the phase associated with the transformations $T^{\frac{1}{h}}$ and $S T^{n} S$. The leading term of $T_{g}(\tau)$ is always $q^{-1}$. Hence $T^{\frac{1}{h}}$ can only act invariably up to a phase by

$$
T^{\frac{1}{h}}\left(T_{g}(\tau)\right)=e^{-2 \pi i / h} T_{g}(\tau)
$$

Suppose that $T_{g}$ is $W_{N}$ invariant. Then we have

$$
\begin{aligned}
S T^{n} S\left(T_{g}(\tau)\right) & =S T^{n}\left(T_{g}(\tau / N)\right) \\
& =e^{-2 \pi i / h} T_{g}(\tau), \quad W_{N} \in \Gamma_{g},
\end{aligned}
$$


using (3.18). Alternatively, from (3.17), $\phi_{g}$ is inverted by $W_{N}$. Assuming $T_{g}$ is $S T^{n} S$ invariant up to a phase implies that for $h \neq 1$ we must have $T_{g}(0)=0$ and

$$
\begin{aligned}
S T^{n} S\left(T_{g}(\tau)\right) & =S T^{n}\left(\frac{c}{T_{g}(\tau / N)}\right) \\
& =e^{2 \pi i / h} T_{g}(\tau), \quad W_{N} \notin \Gamma_{g} .
\end{aligned}
$$

The phase for any element of $\Gamma_{0}(n \mid h)$ is generated by these phases ${ }^{1}$. The phase associated with any $\mathrm{AL}$ transformation $w_{e}$ for $\Gamma_{0}(n \mid h)$ is discussed in the next section.

\section{Moonshine from Orbifolds}

Having outlined the Moonshine properties in Sect. 3 we will now describe a new physical interpretation and explanation for this phenomenon. The correct physical setting turns out to be an orbifold model constructed from the Monster Module $V^{\natural}$ by factoring with a subgroup of $M$. This was suggested in [8] where it was observed that the Thompson series $T_{g}(\tau)$ can be interpreted as an orbifold contribution from untwisted states in $V^{\natural}$ but twisted in the "time direction" by $g$. This suggestion was motivated by Norton's generalised Moonshine formulation [20] which we will return to briefly in Sect. 6. In the notation of Sect. 2 we have

$$
T_{g}(\tau)=\operatorname{Tr}_{V^{\natural}}\left(g q^{L_{0}}\right)=g \underset{1}{\square} \text {. }
$$

This contributes to the partition function for an orbifold based on any group containing $g$.

As we reviewed in Sect. 2, the modular invariance of an orbifold partition function requires the introduction of twisted Hilbert spaces. Thus an $S$ transformation on (4.1) interchanges world-sheet space and time boundary conditions to give

$$
S: g \underset{1}{\square}=1 \underset{g}{\square},
$$

i.e. the partition function for the twisted Hilbert space $H_{g}$ of states periodic in the space direction up to $g$. We will assume that the states are graded by some appropriate Virasoro Hamiltonian $L_{0}$ (according to the usual assumptions of CFT). An explicit general construction of $H_{g}$ is still lacking although we can expect that generalised Kac-Moody algebras have an important role here [5]. Orbifold constructions $[6,7]$ are usually based on an automorphism group of the embedding space of the string (e.g. the Monster Module itself is a $Z_{2}$ quotient of the torus $T^{24}=R^{24} / \Lambda$ ). This embedding allows one to construct a twisted Hilbert space in a natural way. In addition, there is a natural automorphism group induced on this Hilbert space. However, in the present case an element $g$ of $M$ is not in general induced by a geometrical action on the orbifold $T^{24} / Z_{2}$. Therefore, the usual route to constructing twisted Hilbert spaces cannot be taken. An important exception to this is the subgroup of $M$ given by Leech lattice automorphisms which we discuss in Sect. 5.

${ }^{1}$ In [3] and [23] the phases (3.19) and (3.20) appear to have been mistakenly interchanged 
In the absence of a general construction, we will postulate the existence and properties of $H_{g}$ that can be expected from physical arguments. Let $|E\rangle_{g}^{i} \in H_{g}$, where $E$ is the (energy) level of $L_{0}$, the Virasoro Hamiltonian for $H_{g}$ and $i$ labels states within each level. $L_{0}$ contains some normal ordering constant giving the vacuum energy of $H_{g}$. We will assume that for each $E$, the set of states $\left\{|E\rangle_{g}^{i}\right\}$ form a reducible, unitary representation for $C(g)=\{h \in M \mid g h=h g\}$. The states of $H_{g}$ will then contribute to an orbifold based on any abelian subgroup of $M$ containing $g$.

Suppose that $g$ is of order $n$. Then the twisting of a typical state $|E\rangle_{g}^{i}$ of $H_{g}$ is expressed by

$$
g^{i j} e^{2 \pi i L_{0}}|E\rangle_{g}^{j}=e^{2 \pi i \phi_{g} / n}|E\rangle_{g}^{i},
$$

where $g^{i j}$ is shorthand for the appropriate representation of $M$ at level $E$ and $2 \pi \phi_{g} / n$ is a phase which can in general arise as discussed below. Note that by acting with $h \in M$ on (4.3) we see that the twisted space $H_{g}$ is isomorphic to $H_{g^{\prime}}$, where $g^{\prime}=h g h^{-1}$, i.e. $g^{\prime}$ is conjugate to $g$.

The phase in (4.3) is responsible for any global anomalies associated with the modular stability group of an orbifold partition function discussed previously in Sect. 2 [14]. This is clear on considering a $T$ transformation on the partition function $\operatorname{Tr}_{\boldsymbol{H}_{\boldsymbol{g}}}\left(h q^{L_{0}}\right)$ for $h \in M$

$$
T: h \square_{g}=e^{2 \pi i \phi_{g} / n} h g^{-1} \square_{g}
$$

Assuming that the action of $g$ in $H_{g}$ is also of order $n$ then transforming by $T^{n}$ leaves the boundary conditions fixed but introduces a global phase anomaly $e^{2 \pi i \phi_{g}}$. Therefore, the partition function $h \underset{g}{\square}$ does not have a well-defined phase associated with a given pair of boundary conditions. (Note that for a left-right symmetric bosonic string such phase anomalies are irrelevant since the opposite phase appears from the right-hand part. Only in the context of an asymmetric bosonic or heterotic orbifold do such considerations place constraints on the orbifold automorphism group $[14,15])$. This anomalous behaviour can be directly seen in (4.3) in terms of the levels $E$. Applying the twisting operation $n$ times we find

$$
n E=\phi_{g} \bmod 1 \text {. }
$$

When the global phase vanishes $(\bmod 1)$ we get the usual consistency condition on the levels of $L_{0}[14,7,15]$ i.e. $E \in Z / n$. In general, we can express $\phi_{g}$ from $(4.5)$ in terms of the lowest vacuum energy $E_{0}=\phi_{g} / n$.

In [14] the phase associated with a modular transformation of an orbifold trace contribution is discussed. One can show that under a general modular transformation $T_{g}$ becomes

$$
\left(\begin{array}{ll}
a & b \\
c & d
\end{array}\right): g \square=e_{1}^{2 \pi i c d E_{0}} g^{-d} \underset{g^{c}}{\square} .
$$

The phase computed in [14] is for a lattice automorphism. We will assume here that (4.6) is also valid for our orbifold construction.

Let us now consider initially a group element $g$ of order $n$ without any phase anomaly so that $E_{0} \in Z / n$. The more general situation will be discussed later on. The associated Thompson series $T_{g}$ is therefore $\Gamma_{0}(n)$ invariant without phases as follows

$$
\left(\begin{array}{cc}
a & b \\
c n & d
\end{array}\right): g \square=g^{d} \square_{1}=g \square,
$$


since for $(d, n)=1$ we have $T_{g}=T_{g^{d}}$. This follows from the reality of the characteristic equation for each unitary representation of $g$. The invariance of $T_{g}$ under $\Gamma_{0}(n)$ corresponds to part (a) of the Moonshine properties when $h=1$ and $N=n$. Of the 171 Thompson series, 120 are of this form. The remaining series with $h \neq 1$ which do have a global phase anomaly are dealt with later on.

Let us now turn to the reasons for any AL invariance of $T_{g}$ and the hauptmodul property for the full invariance group $\Gamma_{g}=\Gamma_{0}(n)+e_{1}, e_{2}, \ldots$ as given in part (b) of the Moonshine properties. We will show that this will follow from the specific nature of any tachyonic singularities present in the twisted sectors $H_{g^{k}}$. Consider $T_{g}(\tau)$ as a function on the fundamental region $\mathscr{F}_{n}=H / \Gamma_{0}(n)$. The definition (3.5) implies that $T_{g}$ can be singular only at the parabolic cusps of $\mathscr{F}_{n}$ contained in $Q \cup\{i \infty\}$ which are described in the Appendix. There we state and prove a lemma in which a convenient set of inequivalent parabolic cusps for $\Gamma_{0}(n)$ is determined. Consider next the action of $\varrho \in \mathscr{N}(n)$ on $\mathscr{F}_{n}$. Let $\tau_{1} \sim \tau_{2}$ denote the equivalence relation: $\tau_{1}=\gamma \tau_{2}$ for $\gamma \in \Gamma_{0}(n)$. Then $\varrho \tau_{1} \sim \varrho \tau_{2}$ so that an automorphism is induced on $\mathscr{F}_{n}$. In particular, all the parabolic cusps are mapped into each other by $\varrho$. Of particular importance is the cusp point $\tau_{e}$ generated by the action of an AL transform $W_{e}$ of $\Gamma_{0}(n)$ on $i \infty$ :

$$
\begin{aligned}
\tau_{e}=W_{e}(i \infty)=\frac{a e}{c n} & \sim \infty, & e=1, \\
& \sim 0, & e=n, \\
& \sim \frac{1}{f}, \quad & e \neq 1, n,
\end{aligned}
$$

where $f=n / e,(e, f)=1$. We refer to $\tau_{e}$ as an AL cusp from now on. From the Appendix we see that $\tau_{e}$ of (4.8) is amongst the list given. The full set of AL cusps $\left\{i \infty, 0, \tau_{e_{1}}, \tau_{e_{2}}, \ldots\right\}$ is mapped into itself under any AL transformation for $\Gamma_{0}(n)$ by the closure property (3.12). Thus

$$
W_{e_{1}}\left(\tau_{e_{2}}\right)=\tau_{e_{3}}, \quad e_{3}=\frac{e_{1} e_{2}}{\left(e_{1}, e_{2}\right)^{2}}
$$

This closure property is important in proving AL invariance for $T_{g}$. In general, the complete set of parabolic cusps of $\mathscr{F}_{n}$ can be generated by acting on $i \infty$ with the remaining elements of $\mathscr{N}(n)$.

Physically, we see from (4.6) that the behaviour of $T_{g}$ at these cusp points is determined by the presence of tachyonic states in the twisted sectors. Consider any rational point $a / c$ with $(a, c)=1$. Then we can find integers $b, d$ such that $a d-b c=1$. These integers form the components of an element $\gamma$ of $\Gamma$ which maps $\tau=i \infty$ to $a / c$. Therefore, the behaviour of $T_{g}$ at $a / c$ is given by the behaviour of the trace over the twisted sector $H_{g^{c}}$ at $\tau=i \infty$ according to (4.6). This is singular when $L_{0}$ has negative vacuum energy, i.e. $H_{g^{c}}$ contains a tachyonic state. The behaviour of $T_{g}$ at one of the AL cusps $\tau_{e}$ of (4.8) is therefore determined by the presence of a tachyon in the sector twisted by $g^{f}$ of order $e$ [which must also present in the sector twisted by $g^{c f}$, where $\left.(c f, e)=1\right]$. The behaviour at the remaining cusps, associated with the other normalizers, is determined by sectors twisted by $g^{k}$ of order $n^{\prime}=n /(n, k)$, where $\left(n^{\prime}, k\right) \neq 1$. An explicit construction of the twisted sectors would reveal whether or not the vacuum energy is tachyonic. We will postulate the nature of the vacuum energy below and confirm these postulates for some elements of $M$ given 
by Leech lattice automorphisms in Sect. 5. Specifying these singularities will allow us to prove the hauptmodul property for an appropriate fixing group $\Gamma_{g}$.

Consider next the action of an AL transformation $W_{e}$ on $T_{g}(\tau)$. We can relate this to a twisted sector trace by observing that

$$
\begin{aligned}
W_{e} & =\left(\begin{array}{cc}
a e & b \\
c n & d e
\end{array}\right)=\gamma\left(\begin{array}{ll}
e & 0 \\
0 & 1
\end{array}\right), \\
\gamma & =\left(\begin{array}{cc}
a & b \\
c f & d e
\end{array}\right),
\end{aligned}
$$

where $\gamma \in \Gamma$ because det $W_{e}=e$. Acting with $W_{e}$ on $T_{g}$ and applying (4.6) we find that the phase factor is one with

$$
T_{g}\left(W_{e}(\tau)\right)=g_{\mathbf{g}^{-d e}}^{-\square_{f}}(e \tau),
$$

where the right-hand side denotes a trace over $H_{g^{c f}}$ followed by a rescaling of $\tau$ by $e$. (This relation is a generalisation of the fact that the Fricke involution $W_{n}: \tau$ $\rightarrow-1 / n \tau$ is an $S$ transform followed by a rescaling with $n$.) Note that the right-hand side is also $\Gamma_{0}(n)$ invariant since $W_{e}$ is in $\mathcal{N}(n)$.

The set of matrices $W_{e}$ for a given $e$ form a single coset of $\Gamma_{0}(n)$ in $\mathscr{N}(n)$. Therefore, (4.11) is the same for all $c, d$ provided $\operatorname{det} W_{e}=e$. This is equivalent to the condition

$$
(d e, c f)=1 .
$$

We can choose for convenience the $W_{e}$ coset representative with $a=c=1$ for $e \neq n$ and $a=0, c=1$ for $e=n$ in (4.10). These choices correspond to the AL cusp representatives in (4.8). Therefore, $W_{e}$ acting on $T_{g}$ is equivalent to a twisted sector $H_{g^{f}}$ contribution with $\tau$ rescaled by $e$. This in turn is equivalent to a $H_{g^{c s}}$ trace where $(c, e)=1$. From (4.12) we note that $g^{c f}$ is of order $e$ so that the unscaled trace in (4.11) should be $T^{e}$ invariant. This is indeed the case since (4.11) is $T$ invariant.

The property expressed by (4.11) for AL transformations is unique to these elements of the $\mathcal{N}(n)$ in the following sense. If $\varrho \in \mathcal{N}(n)$ can be expressed as a $\Gamma$ transformation followed by a general linear transformation $(\tau \rightarrow A \tau+B)$ then one can show that the cusp $\tau_{e}$ must be an AL cusp. Thus the AL cusps have a special relation to twisted sector contributions.

The final stage of the argument is to specify the nature of any tachyons in the twisted sectors and thereby prove the hauptmodul property. We will demonstrate this initially for $T_{g}$ a $\Gamma_{0}(p)$ invariant for $p$ prime. The fundamental region $\mathscr{F}_{p}$ has only two inequivalent cusps at $i \infty$ and 0 which are interchanged by the unique normaliser $W_{p}$. Applying (4.11) we find

$$
W_{p}: T_{g}(\tau)=1 \square(p \tau)
$$

As discussed in Sect. 3 part (b) of the Moonshine properties implies that either (a) $T_{g}(\tau)$ is inverted by $W_{p}$ in the sense of (3.17) or (b) $T_{g}(\tau)$ is $W_{p}$ invariant. Expanding (4.13) in $q$ we find this implies

$$
\begin{aligned}
1 \underset{g}{1}(p \tau) & =T_{g}(0)+c q+\ldots, \quad W_{p} \notin \Gamma_{g}, \\
& =\frac{1}{q}+0+\ldots, \quad W_{p} \in \Gamma_{g},
\end{aligned}
$$


where $c$ is the constant in (3.17). Therefore, the twisted sector $H_{g}$ either has (a) nonnegative vacuum energy $E_{0}$ or (b) negative vacuum energy $E_{0}=-1 / p$ with degeneracy 1 . The second singular case corresponds to a tachyon in $H_{g}$. We now show that these vacuum energy conditions are sufficient to ensure that $T_{g}$ is a hauptmodul for either (a) $\Gamma_{0}(p)$ or (b) $\Gamma_{0}(p)+$. For case (a) this is immediate since $T_{g}(\tau)$ is regular at the cusp $\tau=0$. Therefore, $T_{g}$ is $\Gamma_{0}(p)$ invariant with a unique simple pole at $q=0$. Applying the Hauptmodul Theorem of Sect. 2 implies that $T_{g}$ is a hauptmodul for $\Gamma_{0}(p)$.

Consider now case (b) where $H_{g}$ contains a tachyon of energy $-1 / p$. Then by (4.14b) we have $T_{g}\left(W_{p} \tau\right)=q^{-1}+\ldots$ and so the function

$$
\psi(\tau)=T_{g}(\tau)-T_{g}\left(W_{p}(\tau)\right)
$$

is regular at both $\tau=0$ and $\tau=i \infty$. The vacuum energy and degeneracy must be as given to ensure this. The function $\psi$ is $\Gamma_{0}(p)$ invariant defining a holomorphic function on $\overline{\mathscr{F}}_{p}$ and therefore must be constant. Acting with $W_{p}$ again shows that $\psi=0$. Therefore, $T_{g}(\tau)$ is invariant under $\Gamma_{0}(p)+$. The cusps at 0 and $i \infty$ are identified under $W_{p}$ and so $T_{g}(\tau)$ has a unique simple pole on $H / \Gamma_{0}(p)+$. By the Hauptmodul Theorem we again find that $T_{g}$ is a hauptmodul for $\Gamma_{0}(p)+$.

These arguments easily generalise to the case of $T_{g}$ a $\Gamma_{0}(n)$ invariant for $n$ not prime. We must specify the vacuum energy of each twisted sector $H_{g^{k}}$ to reproduce the Moonshine properties as follows:

Vacuum Conjectures. Suppose that $H_{g}$ has no global phase anomalies so that $T_{g}$ is $\Gamma_{0}(n)$ invariant with $g$ of order $n$. Let $n^{\prime}=n /(n, k)$ denote the order of $g^{k}$.

I. The vacuum energy $E_{0}$ for $H_{g^{k}}$ is non-negative for $\left(n^{\prime}, k\right) \neq 1$. If $\left(n^{\prime}, k\right)=1$ then $E_{0}$ is either (a) non-negative or (b) negative (tachyonic) with energy $-1 / n^{\prime}$ and degeneracy one.

II. (Closure) Let $e_{1} \| n$ and $e_{2} \| n$. If both sectors $H_{g f_{1}}$ and $H_{g f_{2}}$ are tachyonic then the sector $H_{g^{f_{3}}}$ is also tachyonic where $e_{3}=e_{1} e_{2} /\left(e_{1}, e_{2}\right)^{2}$ and $f_{3}=n / e_{3}$.

We now show that these properties are sufficient to ensure that $T_{g}$ is a hauptmodul for an appropriate group $\Gamma_{g}$. Applying I of the Vacuum Conjectures to (4.11) we find that either (a) $T_{g}(\tau)$ is regular at the AL cusp $\tau_{e}$ or (b) $T_{g}\left(W_{e}(\tau)\right)$ $\sim q^{-1}+\ldots$. These conditions coincide with Norton's observation that $\tau_{e}$ is a singularity of $T_{g}(\tau)$ if and only if $T_{g f}(\tau)$ is invariant under the Fricke involution $\tau \rightarrow-1 / e \tau[20]$. The closure condition II is required to give invariance under more than one AL transformation. Thus if $T_{g}$ is singular at $\tau_{e_{1}}$ and $\tau_{e_{2}}$ it is also singular at $\tau_{e_{3}}$. In general, the singularities of $T_{g}$ are given by $\left\{\tau_{e_{1}}, \tau_{e_{2}}, \ldots\right\}$ a subset of all AL cusps which closes under the corresponding $\mathrm{AL}$ transformations $W_{e_{1}}, W_{e_{2}}, \ldots$ by (4.9).

We may now repeat the argument above for $n=p$. Suppose that $T_{g}$ is singular at $\tau_{e_{1}}$ and consider $\psi(\tau)=T_{g}(\tau)-T_{g}\left(W_{e_{1}}(\tau)\right)$. Clearly, $\psi$ is regular at $\tau=i \infty$ and $\tau_{e_{1}}$ by $\mathrm{I}(\mathrm{a})$ of the Vacuum Conjectures. Likewise, for any other singular AL cusp $\tau_{e_{2}}$ we have $W_{e_{1}}\left(\tau_{e_{2}}\right)=\tau_{e_{3}}$ which by II is also a singular cusp. Therefore, $\psi(\tau)$ is regular also at $\tau_{e_{2}}$. Notice that both the degeneracy and order of the poles must be as given to ensure this. Hence $\psi$ is holomorphic on $\overline{\mathscr{F}}_{n}$ and is therefore a constant. Applying $W_{e_{1}}$ again to $\psi$ shows that $\psi=0$ and so $T_{g}(\tau)$ is $W_{e_{1}}$ invariant. This is similarly true for any other singular $\mathrm{AL}$ cusp. Therefore, $T_{g}(\tau)$ is invariant under $\Gamma_{g}=\Gamma_{0}(n)+e_{1}, e_{2}, \ldots$ if it is singular at $\left\{\tau_{e_{1}}, \tau_{e_{2}}, \ldots\right\}$. This concurs with part (a) of the Moonshine properties for $h=1, N=n$. The associated fundamental region $\overline{\mathscr{F}}_{g}$ has 
at least one cusp at $i \infty$ which is identified with $\left\{\tau_{e_{1}}, \tau_{e_{2}}, \ldots\right\}$ under the corresponding AL transformations. $T_{g}$ has a unique simple pole at this cusp and therefore, $T_{g}$ is a hauptmodul for the genus zero group $\Gamma_{0}(n)+e_{1}, e_{2}, \ldots$ by the Hauptmodul Theorem in agreement with part (b) of the Moonshine properties as required.

Let us return to the remaining Thompson series for which the twisted sector $H_{g}$ has a global phase anomaly as in (4.3). These series are expected to be invariant up to a phase of order $h$ under $\Gamma_{0}(n \mid h)+e_{1}, e_{2}, \ldots$ with $h|24, h| n$ according to the Moonshine properties. We find it necessary to introduce further assumptions to reproduce this result. We will assume that a global phase anomaly can only occur when $T_{g}$ is invariant under $T^{\frac{1}{h}}$ as in (3.18). Therefore, only every $h^{\text {th }}$ level of $V^{\natural}$ contributes. We will also assume that $h \mid n$ and $h \mid 24$. Lastly, we will assume that the global phase anomaly for $H_{g}$ is $\phi_{g}=\frac{l}{h}$ for some integer $l$. Therefore, from (4.4) we have

$$
S T^{n} S: g \square=e^{2 \pi i l / h} g \square
$$

The assumption that $h \mid 24$ is not unreasonable when one considers that the normal ordering constant for $L_{0}$ is typically $-C / 24$ for a twisted space associated with a lattice automorphism for integral central change $C$. From (4.6) it is also clear that $T_{g}$ is $\Gamma_{0}(N)$ invariant without any phases for $N=n h$. Altogether, with the above assumptions we see that $T_{g}$ is $\Gamma_{0}(n \mid h)$ invariant up to phases of order $h$. The invariance group without phases contained in this will be denoted by $\Gamma_{g}^{\prime}$ [which contains $\left.\Gamma_{0}(N)\right]$.

We will now reinterpret $T_{g}(\tau)$ as an orbifold contribution arising from a theory defined on a new world-sheet torus: $w \sim w+1 \sim w+\tau^{\prime}$, where $\tau^{\prime}=h \tau$. On this new torus we may apply the methods employed above to obtain AL invariance and the hauptmodul property. Since $T_{g}(\tau)$ is $T^{\frac{1}{h}}$ invariant up to a phase $e^{-2 \pi i / h}$ only the states at level $E=k h-1$ contribute for $k=0,1,2, \ldots$. Denoting these states by $\left|k^{\prime}\right\rangle_{1}^{i}$ we find that

$$
e^{2 \pi i L_{0}}\left|k^{\prime}\right\rangle_{1}^{i}=e^{-2 \pi i / h}\left|k^{\prime}\right\rangle_{1}^{i},
$$

where $L_{0}^{\prime}=L_{0} / h$. We may thus regard $\left|k^{\prime}\right\rangle_{1}^{i}$ as an untwisted state with a global phase anomaly $e^{-2 \pi i / h}$ and with Virasoro Hamiltonian $L_{0}^{\prime}$. The transformation (4.17) represents the periodicity of $\left|k^{\prime}\right\rangle_{1}^{i}$ under $T^{\prime}: \tau^{\prime} \rightarrow \tau^{\prime}+1$, i.e. under $T^{\frac{1}{h}}$. Referring to Fig. 2 we can see that this corresponds to a modular transformation

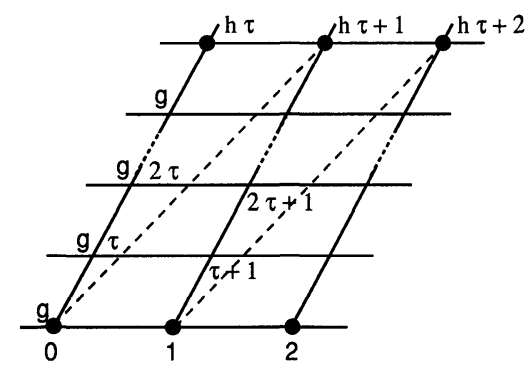

Fig. 2. The world sheet torus is determined by the lattice generated by 1 and $\tau$. The new lattice generated by 1 and $\tau^{\prime}$ is invariant under $\tau^{\prime}=h \tau \rightarrow \tau^{\prime}+1$ 
on a new torus $w \sim w+1 \sim w+\tau^{\prime}$. In addition, the boundary condition in the space direction is now $g^{h}$ for a boundary condition of $g$ on the original torus. Therefore, the trace $T_{g}(\tau)$ is equivalent to a trace of $g^{h}$ over $\left|k^{\prime}\right\rangle_{1}^{i}$ with $L_{0}^{\prime}$ as above on the new torus:

$$
\begin{aligned}
T_{g}(\tau) g & \square \\
& =g^{h} \square_{1}^{\prime}\left(\tau^{\prime}\right) \equiv T_{g^{h}}^{\prime}\left(\tau^{\prime}\right)
\end{aligned}
$$

where the prime denotes the new torus trace. Applying (4.16) we find that $T_{g^{h}}^{\prime}\left(\tau^{\prime}\right)$ is $S^{\prime} T^{\prime} \frac{n}{h} S^{\prime}$ invariant up to the phase $e^{2 \pi i l / h}$. In general, $T_{g^{h}}^{\prime}$ is $\Gamma_{0}\left(\frac{n}{h}\right)$ invariant up to
phases.

On the primed torus the sector twisted by $g^{h}$ has a global phase anomaly $\phi_{g^{n}}^{\prime}=l / h$. From (4.5) we see that the levels $E^{\prime}$ of $L_{0}^{\prime}$ in this sector therefore obey $E^{\prime}=(l+h k) / n$ for $k \in Z$. Each state of $L_{0}^{\prime}$ corresponds to a state on the original torus with $L_{0}=h L_{0}^{\prime}$ and level

$$
E=h E^{\prime}=(l+h k) \frac{h}{n} .
$$

Therefore, from (4.5), since $g^{h}$ is of order $n / h$, these twisted states have no global phase anomaly on the original torus. This observation will allow us to apply the Vacuum Conjectures to sectors twisted by $g^{h}$ and related sectors. This will be sufficient to prove that $T_{g}$ is a hauptmodul for an appropriate fixing group $\Gamma_{g}$.

Consider the action of an AL transformation $w_{e}$ of $\Gamma_{0}(n \mid h)$ as in (3.13) on $T_{g}(\tau)$. Then $\tau^{\prime}=h \tau$ becomes

$$
h w_{e}(\tau)=\frac{a e(h \tau)+b}{c \frac{n}{h}(h \tau)+d e}=W_{e}^{\prime}\left(\tau^{\prime}\right)
$$

where $W_{e}^{\prime}$ is an $\mathrm{AL}$ transformation for $\Gamma_{0}\left(\frac{n}{h}\right)$. Now we can apply the decomposition (4.10) to $W_{e}^{\prime}$ to find that

$$
\begin{aligned}
T_{g}\left(w_{e}(\tau)\right) & =T_{g^{h}}^{\prime}\left(W_{e}^{\prime}\left(\tau^{\prime}\right)\right) \\
& =e^{i \phi} g^{-h d e} \square_{g^{h c f}}^{\prime}\left(e \tau^{\prime}\right),
\end{aligned}
$$

where $f=n / e h,(e, f)=1$ and $e^{i \phi}$ is some phase of order $h$ that depends on the components of $w_{e}$. The trace is over a sector twisted by $g^{\text {hcf }}=g^{n c / e}$ of order $e$ with $(d e, c f)=1$ as before in (4.12). Since $W_{e}^{\prime}$ is in the normaliser of $\Gamma_{0}\left(\frac{n}{h}\right)$ we expect invariance up to phase under $T^{\prime}$ which is consistent with the order of the twisting being $e$. Likewise, $w_{e} \in \mathcal{N}(N)$ and $T_{g}$ is $\Gamma_{0}(N)$ invariant so that we expect $T=T^{\prime h}$ invariance without a phase. This confirms that $e^{i \phi}$ is of order $h$.

We may now apply a similar argument as given previously. Any singular behaviour of $T_{g}$ at the AL cusp $w_{e}(i \infty)$ is due to the presence of a tachyon in the primed torus sector twisted by $g^{n / e}$ [and therefore also $g^{n c / e}$ for $(c, e)=1$ ]. From (4.21) it is clear that $T_{g}$ can only be $w_{e}$ invariant up to a phase if the vacuum energy for the primed torus sector twisted by $g^{n / e}$ is $-1 / e h$. Therefore, from (4.19) we see that the sector twisted by $g^{n / e}$ on the original torus contains a tachyon of energy 
$-1 / e$. This concurs with the Vacuum Conjectures applied to $g^{h}$, which we have argued has no global phase anomaly on the original torus, where we consider the sector twisted by $\left(g^{h}\right)^{n / e h}=g^{n / e}$ of order $e$ with $e \| n / h$. The singularities of $T_{g}$ are therefore supplied also by the Vacuum Conjectures via (4.19) and (4.21). We now show that $T_{g}$ is a hauptmodul for some appropriate group.

It is convenient to consider the modular function

$$
\psi_{g}(\tau)=\left[T_{g}(\tau / h)\right]^{h}
$$

This is $\Gamma_{0}\left(\frac{n}{h}\right)$ invariant without phases. Applying the Vacuum Conjectures for $g^{h}$ we have that either (a) $\psi_{g}\left(W_{e}(\tau)\right)=c+\ldots$ or (b) $\psi_{g}\left(W_{e}(\tau)\right)=q^{-1}+\ldots$, where $W_{e}$ is a $\Gamma_{0}\left(\frac{n}{h}\right) \mathrm{AL}$ transformation. We may then argue that $\psi_{g}$ is $W_{e}$ invariant for every singular AL cusp of $T_{g}(\tau)$ as before. However, the invariance group for $\psi_{g}$ is exactly that for the hauptmodul $T_{g^{h}}(\tau)$ because the singularities arise from the same twisted sectors. Since they both have a simple pole at $q=0$ we have

$$
\left[T_{g}(\tau / h)\right]^{h}=T_{g^{h}}(\tau)+c
$$

for some constant $c$. This is the so-called Harmonic Formula of Conway and Norton [3] which they derive from the Power Map Formula discussed below.

The invariance of $\psi_{g}$ above implies that $\Gamma_{0}(n \mid h)+e_{1}, e_{2}, \ldots$ is the invariance group of $T_{g}$ up to phases of order $h$. This corresponds to part (a) of the Moonshine properties for $h \neq 1$. The invariance group $\Gamma_{g}$ of $T_{g}$ is a subgroup of this generated by $\Gamma_{g}^{\prime}$ [where $\left.\Gamma_{0}(N) \subseteq \Gamma_{g}^{\prime} \cong \Gamma_{0}(n \mid h)\right]$ and any AL transformation $w_{e}$ which leaves $T_{g}$ invariant. From (3.13) we know that $w_{e}$ is a single coset for $\Gamma_{0}(n \mid h)$ but not for $\Gamma_{g}^{\prime}$. Thus for $\gamma \in \Gamma_{0}\left(\frac{n}{h}\right)$ we can define $w_{e}^{\prime}=w_{e} \gamma$ such that $w_{e}^{\prime}$ and $w_{e}$ act on $T_{g}$ with a different phase, e.g. choose $\gamma=T^{k / h}$ for $k=0,1,2, \ldots$. Nevertheless, the corresponding $W_{e}$ action on $\psi_{g}$ in (4.22) is independent of $\gamma$. Likewise, the singular cusp of $T_{g}$ associated with $w_{e}$ is unique as the choice of $\gamma=T^{k / h}$ indicates. We may therefore choose a representative, denoted by $\hat{w}_{e}$, from the set of matrices $w_{e}$ which has no phase. Then $T_{g}(\tau)$ is invariant under $\Gamma_{g}$ given by adjoining to $\Gamma_{g}^{\prime}$ the appropriate AL transformations $\hat{w}_{e_{1}}, \hat{w}_{e_{2}}, \ldots$ As before, $T_{g}$ is only singular at the corresponding AL cusps which are now identified under $\Gamma_{g}$ so that $T_{g}$ is a hauptmodul for the genus zero group $\Gamma_{g}$.

The Vacuum Conjectures can be further exploited to prove the Power Map Formula of Conway and Norton [3]. This result does not follow from the Moonshine properties but is an independent empirical observation.

Power Map Formula. Suppose $T_{g}$ is invariant up to phases under $\Gamma_{0}(n \mid h)+e_{1}, e_{2}, \ldots$. Then for any $d, T_{g^{d}}$ is invariant up to phases under $\Gamma_{0}\left(n^{\prime} \mid h^{\prime}\right)+e_{1}^{\prime}, e_{2}^{\prime}, \ldots$, where $n^{\prime}=n /(n, d), h^{\prime}=h /(h, d)$ and $e_{1}^{\prime}, e_{2}^{\prime}, \ldots$ are the divisors of $n^{\prime} / h^{\prime}$ amongst the numbers $e_{1}, e_{2}, \ldots$.

Let us prove this initially for $T_{g}$ without a global phase anomaly so that $h=h^{\prime}=1$ above. The general result will then follow from the Harmonic Formula (4.23). Consider $g^{\prime}=g^{d}$ of order $n^{\prime}=n /(n, d)$. Then $T_{g^{\prime}}$ is $\Gamma_{0}\left(n^{\prime}\right)$ invariant since no global phase anomaly occurs for any $g^{k}$ sector. If $T_{g^{\prime}}$ is $W_{e^{\prime}}$ invariant $\left(e^{\prime}\left\|n^{\prime}\right\|\right)$ then the vacuum energy is $-1 / e^{\prime}$ for the sector twisted by $\left(g^{\prime}\right)^{n^{\prime} / e^{\prime}}=\left(g^{n / e^{\prime}}\right)^{d^{\prime}}$ of order $e^{\prime}$, where $d^{\prime}=d /(n, d)$ and $\left(d^{\prime}, e^{\prime}\right)=1$. But $T_{g}$ is singular only at its AL cusps by the Vacuum 
Conjectures and so the sector twisted by $\left(g^{n / e^{\prime}}\right)^{d^{\prime}}$ can only be tachyonic provided $e^{\prime} \| n$ by (4.6). Therefore, $W_{e^{\prime}}$ is an AL symmetry of $T_{g}$ also. On the other hand, if $W_{e}$ is an AL symmetry for $T_{g}$ then the sector twisted by $\left(g^{n / e}\right)^{c}$ of order $e,(c, e)=1$, has vacuum energy $-1 / e$. This sector can only influence the behaviour of $T_{g^{\prime}}$ provided $e \mid n^{\prime}$ (and hence $e \| n^{\prime}$ implying that $T_{g^{\prime}}$ is $W_{e}$ invariant). If $e \nmid n^{\prime}$ then $(e, d) \neq 1$ and so $\left(g^{\prime}\right)^{k}=g^{d k}$ cannot be of order $e$ for any $k$. Therefore, no modular transformation of $T_{g^{\prime}}$ can introduce this tachyonic sector and hence $T_{g^{\prime}}$ cannot be $W_{e}$ invariant. To summarise, we find that if $T_{g}$ is $\Gamma_{0}(n)+e_{1}, e_{2}, \ldots$ invariant then $T_{g^{d}}$ is $\Gamma_{0}\left(n^{\prime}\right)+e_{1}^{\prime}, e_{2}^{\prime}, \ldots$ invariant where $e_{1}^{\prime}, e_{2}^{\prime}, \ldots$ are the divisors of $n^{\prime}=n /(n, d)$ amongst $e_{1}, e_{2}, \ldots$. This corresponds to the Power Map Formula for $h=h^{\prime}=1$.

The general result now follows by using the Harmonic Formula (4.23). We apply the global phase anomaly free Power Map Formula to $T_{g^{n}}$ with power $d^{\prime}=d /(d, h)$. Then $T_{g^{d^{\prime} h}}$ is $\Gamma_{0}(m)+e_{1}^{\prime}, e_{2}^{\prime}, \ldots$ invariant where

$$
m=\frac{\frac{n}{h}}{\left(\frac{n}{h}, d^{\prime}\right)}=\frac{n^{\prime}}{h^{\prime}}
$$

with $n^{\prime}=n /(n, h)$ and $h^{\prime}=h /(h, d)$ and where $e_{1}^{\prime}, e_{2}^{\prime}, \ldots$ are the divisors of $m$ amongst $e_{1}, e_{2}, \ldots$. The relation (4.24) follows from the identity $(n, d)=(h, d)\left(n / h, d^{\prime}\right)$. Finally, noting that $g^{d^{\prime} h}=\left(g^{d}\right)^{h^{\prime}}$ and applying the Harmonic Formula (4.23) we find that $T_{g^{d}}$ is invariant up to phases under $\Gamma_{0}\left(n^{\prime} \mid h^{\prime}\right)+e_{1}^{\prime}, e_{2}^{\prime}, \ldots$ as claimed.

\section{Leech Lattice Automorphisms}

In the last section we derived the Moonshine properties for a Thompson series $T_{g}$ by assuming the Vacuum Conjectures for the associated twisted spaces. In this section we will describe the construction of such spaces for a subgroup of $M$ based on Leech lattice automorphisms and demonstrate the validity of the Vacuum Conjectures in many such cases.

We begin by returning to the untwisted Hilbert space $H_{(+)}$of (2.5). The highest weight state $|\beta\rangle, \beta \in \Lambda$ can be constructed by introducing the vertex operator $V(\beta, z)$ as follows $[1,11]$

$$
\begin{gathered}
V(\beta, z)=: e^{i \beta \cdot x(z)}: c(\beta), \\
|\beta\rangle=V(\beta, 0)|0\rangle,
\end{gathered}
$$

where $c(\beta)$ is an element of the central extension $\hat{\Lambda}$ of $\Lambda$ by $\{ \pm 1\}$ which is required to ensure the associativity of the vertex operator algebra. The coefficients $c(\beta)$ obey the cocycle conditions

$$
\begin{gathered}
c(\alpha) c(\beta)=\varepsilon(\alpha, \beta) c(\alpha+\beta), \\
\varepsilon(\alpha, \beta)=(-1)^{\alpha \cdot \beta} \varepsilon(\beta, \alpha) .
\end{gathered}
$$

Consider $\bar{a}$ an element of the automorphism group of the Leech lattice known as the Conway group $\cdot 0$, i.e. $\bar{a}: \Lambda \rightarrow \Lambda$ and $\bar{a} \alpha \cdot \bar{a} \beta=\alpha \cdot \beta$ for all $\alpha, \beta \in \Lambda$. Each such automorphism induces a natural automorphism on $H_{(+)}$by

$$
\bar{a}:|\beta\rangle \rightarrow|\bar{a} \beta\rangle
$$


with the remaining Fock states (2.5) transforming in an obvious way. This transformation preserves $L_{0}$ and hence the states at each level transform amongst themselves.

From the point of view of the vertex operator (5.1 a) there is a larger class of related automorphisms available. These are the automorphisms of $\hat{\Lambda}$, denoted by $C_{0}$, that preserves (5.2). $C_{0}$ is a central extension by $Z_{2}^{24}$ of $\cdot 0$ denoted by $C_{0}=2^{24}(\cdot 0)$. (This situation is familiar for a Lie algebra of rank $l$ where the group of Weyl reflections $W$ is extended by $Z_{2}^{l}$ to form the discrete automorphism group $\left.Z_{2}^{l}(W)[24]\right)$. For $a \in C_{0}$ we have $[1,20]$

$$
\begin{gathered}
a: c(\beta) \rightarrow(-1)^{f_{a}(\beta)} c(\bar{a} \beta), \\
a:|\beta\rangle \rightarrow(-1)^{f_{a}(\beta)}|\bar{a} \beta\rangle,
\end{gathered}
$$

where $\bar{a} \in \cdot 0$ is associated with $a$ and where $f_{a}(\beta)$ is a $Z_{2}$ central extension of $\bar{a}$ obeying $f_{a}(\alpha+\beta)=f_{a}(\alpha)+f_{a}(\beta)$. It is useful to realise $f_{a}(\alpha)$ as follows. Let $\left\{e^{(r)}\right\}$ be a basis for $\Lambda$ and $\left\{e_{(s)}\right\}$ a dual basis with $e^{(r)} \cdot e_{(s)}=\delta_{s}^{r}$. Then for $\alpha=\alpha_{r} e^{(r)}$ we have $f_{a}(\alpha)=\alpha_{r} m^{r}$ with $m^{r}=f_{a}\left(e^{(r)}\right) \in Z_{2}$. Defining the lattice vector $m=m^{r} e_{(r)}$ we have for any $\alpha \in \Lambda$

$$
f_{a}(\alpha)=e^{i \pi \alpha \cdot m} .
$$

The set of inequivalent choices of $m$ for a given $\bar{a}$ is given by $\Lambda / 2 \Lambda$ of dimension $2^{24}$ giving $C_{0}=2^{24}(\cdot 0)$. The transformation (5.3) then corresponds to the trivial extension $m=0$.

We turn now to the Monster Module $V^{\natural}$ and describe the role played by such automorphisms. We first describe the automorphism group $\hat{C}_{0}$ of the full set of states $H_{(+)} \oplus H_{(-)}$where no mixing between untwisted and twisted sectors occurs. A $Z_{2}$ quotient of $\hat{C}_{0}$ then provides the appropriate group for $V^{\natural}$.

The automorphism group $C_{0}$ for $H_{(+)}$induces an automorphism group on $H_{(-)}$ of (2.14) as follows. Recall that the vacuum states of $H_{(-)}$are associated with the fixed points $\Lambda / 2 \Lambda$ of $g: x \rightarrow-x$. Let $K=\{c(2 \alpha) \mid \alpha \in \Lambda\}$ which is a subgroup of $\hat{\Lambda}$ isomorphic to $2 \Lambda[1,20]$. From $(5.5)$ we have $f_{a}(2 \alpha)=1$ so $K$ is also invariant under $C_{0}$. Therefore, $C_{0}$ induces an automorphism group on $\hat{A} / K \cong 2_{+}^{1+24}$ the "extra special" group of order $2^{25}[1,2]$. This automorphism group has a unique $2^{12}$-dimensional representation acting on a vector space denoted by $T$. The vacuum states $\left\{\left|\sigma_{a}\right\rangle\right\}$ of $H_{(-)}$introduced in Sect. 2 form a set of basis vectors for $T$. The representation acting on $T$ is in fact a projective representation of $C_{0}$ with a two-fold covering $[1,20]$ where defining

$$
\hat{C}_{0}=\left\{\left(a, a_{T}\right) \in C_{0} \mid a_{T}: T \rightarrow T\right\}
$$

we have $C_{0}=\hat{C}_{0} /\left\{I, \pm I_{T}\right\}$ where $I\left(I_{T}\right)$ is the identity of $C_{0}(T)$. The group $\hat{C}_{0}$ is then the automorphism group of $H_{(+)} \oplus H_{(-)}$where no mixing between untwisted and twisted states occurs, i.e. all elements of $\hat{C}_{0}$ commute with $i=\left(I,-I_{T}\right)$ which is the "fermion number" introduced in Sect. 2.

Consider next the $g$ invariant projection $V^{\natural}$ of $H_{(+)} \oplus H_{(-)}$and notice that the element $\left(-I,-I_{T}\right)$ has eigenvalue +1 for all states of $V^{\natural}$ from $(2.9 \mathrm{a})$ and $(2.16 \mathrm{a})$. Therefore, the automorphism group of $V^{\natural}$ that commutes with $i$ is

$$
C=\hat{C}_{0} /\left\{ \pm\left(I, I_{T}\right)\right\}=2_{+}^{1+24}(\cdot 1),
$$

where $\cdot 1=\cdot 0 /\{ \pm 1\}$ is the Conway simple group. The group $C$ is the centraliser of $i$ in $M$. The correct identification of $C$ with $2_{+}^{1+24}(\cdot 1)$ is a crucial part of the Monster 
group construction since Griess has shown that $C$ together with only one other involution element $\sigma$ generates all of $M$ [2]. FLM [1] constructed $\sigma$ (which mixes $H_{(+)}$and $H_{(-)}$) from a triality symmetry [25] inherent in the Monster Module construction to show that $V^{\natural}$ has $M$ as its automorphism group.

FLM have also shown how to compute the Thompson series $T_{g}$ for any $g \in C$. Let $g=\left(a, a_{T}\right) \in C$ corresponding to $\pm\left(a, a_{T}\right) \in \hat{C}_{0}$. We will define the basic modular functions that arise in calculating $\bar{T}_{g}(\tau)$. The matrix $\bar{a} \in \cdot 0$ associated with $a$ has characteristic polynomial

$$
\operatorname{det}(x-\bar{a})=\prod_{k \mid n}\left(x^{k}-1\right)^{a_{k}}
$$

where $\sum k a_{k}=24$ and where each $a_{k}$ is a (not necessarily positive) integer with $\bar{a}$ of order $n$. For any such $\bar{a}$ we can define the generalised eta function

$$
\eta_{\bar{a}}=\prod_{k \mid n}(\eta(k \tau))^{a_{k}}
$$

Likewise, we can define the theta function for $\bar{a}$

$$
\Theta_{\bar{a}}(\tau)=\sum_{\beta \in \Lambda_{\bar{a}}} q^{\beta^{2} / 2}
$$

where $\Lambda_{\bar{a}}=\{\beta \in \Lambda \mid \bar{a} \beta=\beta\}$ is the invariant lattice for $\bar{a}$ of dimension $d=\sum a_{k}$, the number of unit eigenvalues of (5.8). We may also define a modified theta function for $a$ by

$$
\begin{aligned}
\Theta_{a}^{\prime}(\tau) & =\sum_{\beta \in \Lambda_{\bar{a}}}(-1)^{f_{a}(\beta)} q^{\beta^{2} / 2} \\
& =\sum_{\beta \in \Lambda_{\bar{a}}} e^{i \pi \beta \cdot m_{\|}} q^{\beta^{2} / 2}
\end{aligned}
$$

where $m_{\|}$is the projection of $m$ in (5.5) parallel to $\Lambda_{\bar{a}}$, i.e. defining $\mathscr{P}_{\bar{a}}=\left(1+\bar{a}+\ldots+\bar{a}^{n-1}\right) / n$ we have $m_{\|}=\mathscr{P}_{\bar{a}} m$ and $\beta \cdot m=\beta \cdot m_{\|}$for $\beta \in \Lambda_{\bar{a}}$.

We may now compute the Thompson series for $g$ by expanding the partition function as

$$
\begin{aligned}
g \underset{1}{g}= & \frac{1}{2}[a \square+-a \square] \\
& +\frac{1}{2}\left[\left(a, a_{T}\right) \square+\left(-a,-a_{T}\right) \square\right],
\end{aligned}
$$

where the right-hand side corresponds to traces over $H_{(+)}$and $H_{(-)}$. Each of these traces can be separately computed from the Fock spaces $(2.5)$ and $(2.14)$ to obtain the closed form [1]

$$
\begin{aligned}
T_{g}(\tau)= & \frac{1}{2}\left[\frac{\Theta_{a}^{\prime}(\tau)}{\eta_{\bar{a}}(\tau)}+\frac{\Theta_{-a}^{\prime}(\tau)}{\eta_{-\bar{a}}(\tau)}\right] \\
& +\frac{1}{2}\left[\operatorname{Tr}\left(a_{T}\right) \frac{\eta_{\bar{a}}(\tau)}{\eta_{\bar{a}}(\tau / 2)}+\operatorname{Tr}\left(-a_{T}\right) \frac{\eta_{-\bar{a}}(\tau)}{\eta_{-\bar{a}}(\tau / 2)}\right],
\end{aligned}
$$

where $\operatorname{Tr}\left( \pm a_{T}\right)$ is a trace over the representation space $T$.

As described in Sect. 4, we expect $T_{g}(\tau)$ to be $\Gamma_{0}(n)$ invariant up to a global phase. Notice that we can also consider the first trace of (5.12) as a new Thompson series for the untwisted sector $H_{(+)}$which we denote by $T_{a}^{(+)}=a \square$. Naively, it would appear that $T_{a}^{(+)}$is also $\Gamma_{0}(n)$ invariant up to a phase. However, it is necessary to check that in the sector twisted by $a$, the automorphism $\hat{a}$ induced by $a$ is also of 
order $n$. This is in general not the case as we shall see below. To understand this point it is useful to consider the modular properties of $\eta_{\bar{a}}$ and $\Theta_{a}^{\prime}$ before describing the twisted sector construction.

Suppose that $h \mid k$ for all $a_{k} \neq 0$ then $\eta_{\bar{a}}$ is $T^{\frac{1}{h}}$ invariant up to a phase $e^{2 \pi i / h}$ with $h|n, h| 24$. Under $S$ we find

$$
\eta_{a}(-1 / \tau)=\frac{(-i \tau)^{d / 2}}{D^{1 / 2}} \prod_{k \mid n}(\eta(\tau / k))^{a_{k}}
$$

where

$$
D=\prod_{k \mid n} k^{a_{k}}
$$

The modular function (5.14) is covariant under $T^{n}$ up to a phase $e^{-2 \pi i n E_{0}}$, where

$$
E_{0}=-\frac{1}{24} \sum_{k \mid n} \frac{a_{k}}{k}
$$

which follows directly from the leading behaviour $q^{-E_{0}}$ of the product of eta functions. These properties are clearly reproducing some of the features of the global phase anomaly $\phi_{g}=n E_{0}$ of (4.3) for vacuum energy $E_{0}$. In particular, we note that $\phi_{g}=l / h^{\prime}$ for integers $l, h^{\prime}$ where $h^{\prime} \mid 24$. At this stage $h$ and $h^{\prime}$ are not yet related.

Consider next the theta functions $\Theta_{\bar{a}}$ and $\Theta_{a}^{\prime}$. In many cases these are actually equivalent. Thus for $n$ odd we have $f_{a}(\beta)=0 \bmod 2$ for all $\beta \in \Lambda_{\bar{a}}$ and $\Theta_{\bar{a}}=\Theta_{a}^{\prime}$. Alternatively, if $n$ is even and $a=b^{2}, b \in C_{0}$ then $f_{a}(\beta)=f_{b}(\beta) \pm f_{b}(\beta)=0 \bmod 2$ for $\beta \in \Lambda_{\bar{a}}, b \beta= \pm \beta$ and again $\Theta_{\bar{a}}=\Theta_{a}^{\prime}$. In general, the modular properties of $\Theta_{a}^{\prime}$ are as follows. If $h \mid \beta^{2} / 2$ for all $\beta \in \Lambda_{a}$ then $\Theta_{a}^{\prime}$ is $T^{1 / h}$ invariant. Under $S$ we have

$$
\Theta_{a}^{\prime}(-1 / \tau)=\frac{(-i \tau)^{d / 2}}{V_{\bar{a}}} \Theta_{a}^{\prime *}(\tau)
$$

using Poisson resummation $[11,10]$, where $V_{\bar{a}}$ is the lattice volume $\left.\left|\Lambda_{\bar{a}}^{*}\right| \Lambda_{\bar{a}}\right|^{1 / 2}$ and $\Theta_{a}^{\prime *}$ is the modified theta function for the dual lattice $\Lambda_{a}^{*}$

$$
\Theta_{a}^{\prime *}(\tau)=\sum_{\beta^{*} \in \Lambda_{a}^{*}} q^{\left(\beta^{*}+m_{\| 1} / 2\right)^{2}} /^{2} .
$$

The dual lattice is in fact equivalent to the parallel projection of $\Lambda$ with $\Lambda_{a}^{*}=\Lambda_{\|} \equiv \mathscr{P}_{a} \Lambda[15]$. Thus (5.18) is invariant under $m_{\|} \rightarrow m_{\|}+2 \beta_{\|}$for $\beta_{\|} \in \Lambda_{\|}$ confirming that the inequivalent choices for $m$ are given by $\Lambda / 2 \Lambda$. In general, the norm of $\beta_{\|} \in \Lambda_{\|}$obeys

$$
\begin{aligned}
& n \beta_{\|}^{2}=0 \bmod 2, \quad n \text { odd, } \\
& n \beta_{\|}^{2}=0 \bmod 1, \quad n \text { even, }
\end{aligned}
$$

which follows from the fact that $n \beta_{\|}=n \mathscr{P}_{\bar{a}} \beta \in \Lambda_{\bar{a}}$.

Consider now the cases for which $m_{\|}=0$ and so $\Theta_{\bar{a}}=\Theta_{a}^{\prime}$. Considering (5.14) and (5.17) we find the $S$ transform of $T_{\bar{a}}^{(+)}$to be

$$
1 \square_{\bar{a}}=\frac{D^{1 / 2}}{V_{\bar{a}}} \frac{\Theta_{\bar{a}}^{*}(\tau)}{\prod(\eta(\tau / k))^{a_{k}}}=\frac{D^{1 / 2}}{V_{\bar{a}}} q^{E_{0}}+\ldots
$$

Then (5.19) implies that $T_{\bar{a}}^{(+)}$is $S T^{n} S$ invariant for $n$ odd but $S T^{2 n} S$ invariant for $n$ even (up to a phase $e^{2 \pi i n E_{0}}$ and $e^{4 \pi i n E_{0}}$, respectively). This latter property reflects the 
fact that in general, the automorphism $\bar{a}$ induced by $\bar{a}$ on the sector twisted by $\bar{a}$, can be of order $2 n$ leading to a $Z / 2 n$ grading rather than the naively expected $Z / n$ grading $[26,15]$. Fortunately, for Leech lattice automorphisms, this problem does not arise for the following reasons. For $n$ even, in general, we have using $\beta \cdot \bar{a}^{k} \cdot \beta$ $=\beta \cdot \bar{a}^{n-k} \cdot \beta$ that $[15]$

$$
n \beta_{\|}^{2}=\beta \cdot \bar{b} \cdot \beta \bmod 2,
$$

where $\bar{b}=\bar{a}^{n / 2}$ is of order 2 . The Conway group contains three conjugacy classes of order 2 [23]. However, two of these classes are members of the same conjugacy class in $M$ which leads, according to [3], to two independent $S T^{2} S$ invariant Thompson series. Hence (5.21) implies that $n \beta_{\|}^{2}=0 \bmod 2$ and therefore, $T_{a}^{(+)}$is $S T^{n} S$ invariant up to the phase $e^{2 \pi i n E_{0}}$ for all $n$. Similarly, the sum of remaining three traces of $(5.12)$ is $S T^{n} S$ invariant up to a phase with $-\bar{a} \square$ separately invariant for $n$ even.

The situation for $m_{\|} \neq 0$ is more complicated. Then we find that $n \beta_{\|} \cdot m_{\|} \in Z$ so that $\Theta_{a}^{\prime}$ is $S T^{2 n} S$ covariant up to the phase $e^{\pi i n m_{\|}^{2} / 4}$. Therefore, the sector twisted by $a$ has $Z / 2 n$ grading. This implies that the induced automorphism $\hat{a}$ in this sector is of order $2 n$ in general. Nevertheless, the combination of traces in (5.14) is $S T^{n} S$ invariant up to phase which presumably results from a transformation of the form

$$
\begin{aligned}
S T^{n} S[a \square] & =S T^{n}[+\underset{a}{\square}] \\
& =e^{i \phi} S[-\underset{a}{\square}]=e^{i \phi} a \square
\end{aligned}
$$

for some phase $e^{i \phi}$ and similarly for the trace $-a \square$.

Let us restrict ourselves from now on to the case where $m_{\|}=0$ and construct the $\bar{a}$ twisted sector $[6,27,26]$. The starting point is to consider the mode expansion for $x^{i}(z)$ satisfying the twisted boundary condition $x^{i}(z)=\bar{a}^{i j} x^{j}\left(e^{2 \pi i} z\right)+\beta^{i}, \beta \in \Lambda$ as in (2.12). (For $m_{\|} \neq 0$ this geometrical approach of quotienting $T^{24}$ by a lattice automorphism is no longer valid and some different method of construction is required.) It is convenient to express $\bar{a}$ in a diagonal basis with $\bar{a}=\left(\begin{array}{cc}I_{d} & 0 \\ 0 & b\end{array}\right)$, where $I_{d}$ is the $d$-dimensional identity matrix and $b=\operatorname{diag}\left(e^{2 \pi i r_{d+1}}, \ldots, e^{2 \pi i r_{24}}\right)$ for $0<n r_{i}<n$. Defining the projections of $x$ by $x_{\|}=\mathscr{P}_{\bar{a}} x$ and $x_{T}=\left(1-\mathscr{P}_{\bar{a}}\right) x$ we obtain the mode expansion

$$
\begin{aligned}
& x_{\|}^{i}=q_{\|}^{i}-i p_{\|}^{i} \ln z+i \sum_{n \in Z} \frac{\alpha_{n}^{i}}{n} z^{-n}, \quad i=1, \ldots, d, \\
& x_{T}^{i}=q_{T}^{i}+i \sum_{n \in Z+r_{i}} \frac{\alpha_{n}^{i}}{n} z^{-n}, \quad i=d+1, \ldots, 24,
\end{aligned}
$$

where $q_{\|}, p_{\|}$, and $\alpha_{n}$ obey the usual commutation relations (2.1). The vector $q_{T}$ denotes any fixed point of $T^{24}$ under $\bar{a}$. The mode expansion obeys the $\bar{a}$ twisting condition provided

$$
p_{\|} \in \Lambda_{\|}, \quad q_{T} \in(1-b)^{-1} \Lambda_{\bar{a}}^{T},
$$

where $\Lambda_{\bar{a}}^{T}=\left\{\beta \in \Lambda \mid \mathscr{P}_{\bar{a}} \beta=0\right\}$ is the set of lattice vectors perpendicular to $\Lambda_{\bar{a}}$. The set of inequivalent fixed points $q_{T}$ is determined by the coset $\Lambda_{\bar{a}}^{T} /(1-b) \Lambda_{T}$, where $\Lambda_{T}=\left(1-\mathscr{P}_{\bar{a}}\right) \Lambda[15]$. In addition, just as $\Lambda_{\|}=\Lambda_{\bar{a}}^{*}$ so $\Lambda_{T}=\Lambda_{\bar{a}}{ }^{T *}$. It is straightforward 
to show also that $\Lambda_{\bar{a}}^{*} / \Lambda_{\bar{a}}=\Lambda_{\bar{a}}^{T *} / \Lambda_{\bar{a}}^{T}$ so that the lattice volume $V_{\bar{a}}$ is given by $\left|\Lambda_{T} / \Lambda_{\bar{a}}^{T}\right|^{1 / 2}$. Therefore, the number of fixed points $n_{\bar{a}}$ of $\bar{a}$ is [15]

$$
\begin{aligned}
n_{\bar{a}} & =\left|\Lambda_{\bar{a}}^{T} /(1-b) \Lambda_{T}\right| \\
& =V_{\bar{a}}^{-2} \operatorname{det}(1-b)=\frac{D}{V_{\bar{a}}^{2}},
\end{aligned}
$$

where extracting a factor of $(x-1)^{d}$ from (5.9) we obtain $\operatorname{det}(1-b)=\prod k^{a_{k}}=D$ of (5.15). The $n_{\bar{a}}$ fixed points determine the vacuum states $\left|q_{T}\right\rangle$ for the left and right moving theory. The left moving sector is expected from (5.20) to have a vacuum degeneracy of $n_{\bar{a}}^{1 / 2}=D^{1 / 2} / V_{\bar{a}}$ which is always an integer $[15,26]$. Thus there must exist a set of left moving vacuum states $\left\{\left|\sigma_{T}^{r}\right\rangle\right\}$ for $r=1,2, \ldots, n_{\bar{a}}^{1 / 2}$. The full twisted Hilbert space is then constructed as a Fock space by acting with $\alpha_{n}^{i}$ on the highest weight states $\left|\beta_{\|}\right\rangle \otimes\left|\sigma_{T}^{r}\right\rangle, \beta_{\|} \in \Lambda_{\|}$generalising (2.24). The states are graded by the Virasoro Hamiltonian $L_{0}=p_{\|}^{2} / 2+\sum \alpha_{-n}^{i} \alpha_{n}^{i}+E_{0}$, where $E_{0}$ is the normal ordering constant $\frac{1}{2} \sum_{i} \sum_{n}\left(n+r_{i}\right)$ which provides the vacuum energy. By zeta function regularisation or otherwise we find [7]

$$
E_{0}=-1+\frac{1}{4} \sum_{i=1}^{24} r_{i}\left(1-r_{i}\right)
$$

This sum can be performed using the parametrisation (5.8) to obtain

$$
E_{0}=-1+\frac{1}{4} \sum_{k \mid n} a_{k} \sum_{j=1}^{k-1} \frac{j}{k}\left(1-\frac{j}{k}\right)=-1+\frac{1}{24} \sum_{k \mid n} a_{k}\left(k-\frac{1}{k}\right)
$$

which agrees with (5.16) on using $\sum k a_{k}=24$. We may now compute the twisted sector partition function $1 \square_{\bar{a}}$ to obtain the expected result (5.20).

Let us next discuss the validity of the Vacuum Conjectures for the sectors twisted by $\bar{a}^{r}$. We will consider $T_{\bar{a}}^{(+)}$for $\bar{a} \in \cdot 0$ and show that $T_{\bar{a}}^{(+)}$is a hauptmodul for an appropriate modular group given some specific properties of the parameters $a_{k}$ and the invariant lattice $\Lambda_{\bar{a}}$. The remaining terms of (5.12) then sum to form the same hauptmodul differing only by a constant. It was originally proposed by Conway and Norton [3] that $T_{\bar{a}}^{(+)}$is a hauptmodul for all $\bar{a} \in \cdot 0$. However, this conjecture turns out to be false in some cases ${ }^{2}$. The specific reason for this failure in these cases is not known to us.

Consider the parameters $a_{k}$ of (5.8) and suppose that for some $h$ we have that $h \mid k$ for all $a_{k} \neq 0$. Then $\eta_{\bar{a}}$ is $T^{\frac{1}{h}}$ invariant up to a phase $e^{2 \pi i / h}$. Let $e \| \frac{n}{h}$ with $f=n / e h$ and decompose $k$ uniquely for $a_{k} \neq 0$ as follows

$$
k=h k_{e} k_{f}, \quad k_{e}=\left(\frac{k}{h}, e\right), \quad k_{f}=\left(\frac{k}{h}, f\right),
$$

${ }^{2}$ In [20] it is stated that the first counter-example was found by Koike and that all counterexamples have been found by $\mathbf{K}$. Harada and collaborator 
where $\left(k_{e}, k_{f}\right)=1$. The action of the associated AL transformation $w_{e}$ of (3.13) on $\eta_{\bar{a}}$ can now be computed using

$$
\begin{aligned}
h k_{e} k_{f} w_{e}(\tau) & =\gamma\left(h \frac{e}{k_{e}} k_{f} \tau\right), \\
\gamma & =\left(\begin{array}{cc}
a k_{e} & b k_{f} \\
c n / e h k_{f} & d e / k_{e}
\end{array}\right),
\end{aligned}
$$

where $\gamma \in \Gamma$. Therefore, $\eta_{\bar{a}}$ is transformed into itself (up to phases and $c \tau+d$ factors) if the parameters $a_{k}$ obey for all $k$

$$
a_{h k_{e} k_{f}}=a_{h\left(e / k_{e}\right) k_{f}} .
$$

On the other hand, $\eta_{a}$ is inverted by $w_{e}$ if

$$
a_{h k_{e} k_{f}}=-a_{h\left(e / k_{e}\right) k_{f}} \text {. }
$$

This second relation implies that $d=0$ so that $\Lambda_{\bar{a}}=0$ and $T_{a}^{(+)}=1 / \eta_{\bar{a}}$ in this case.

We can now restate Conway and Norton's original conjecture about $T_{a}^{(+)}$as follows: For most conjugacy classes of $\cdot 0$ in $M$, a representative $\bar{a}$ can be chosen for each $e \| \frac{n}{h}$ such that either (5.30) or (5.31) is obeyed.

Notice that the $w_{e}$ inversion property is stronger than the general Moonshine properties. However, in general, within each conjugacy class of $\cdot 0$ in $M$ there are a number of conjugacy classes of $\cdot 0$ in itself, each of which has the same Thompson series up to a constant. The claim is that for some representative choice of $\bar{a}$ within these classes, $T_{a}^{(+)}$is $w_{e}$ inverted for an appropriate constant. This claim is strengthened by the observation that all Thompson series listed in Table 3 of [3], which are not invariant under at least one AL transformation, have the form $T_{\bar{a}}^{(+)}=1 / \eta_{\bar{a}}$ corresponding to $d=0$.

Assuming that (5.30) or (5.31) is true for a choice of $\bar{a}$, we can immediately calculate $E_{0}$ from (5.16) by choosing $e=n / h, f=1$. Then $a_{k}= \pm a_{k^{\prime}}$ for $k^{\prime}=n h / k$ and

$$
E_{0}=\mp \frac{1}{24 n h} \sum_{k^{\prime} \mid n} k^{\prime} a_{k^{\prime}}=\mp \frac{1}{n h}
$$

which agrees with the vacuum energy of Sect. 4 for a global phase anomaly $e^{ \pm 2 \pi i / h}$.

We may also calculate the vacuum energy for powers of $\bar{a}$. Let $\bar{b}=\bar{a}^{r}$ which is of order $n^{\prime}=n /(n, r)$ and which has characteristic equation parameters

$$
b_{k^{\prime}}=\sum_{k=k^{\prime}(k, r)}(k, r) a_{k}
$$

where $k^{\prime} \mid n^{\prime}$. If $(n, r)=1$ then $b_{k}=a_{k}$ and the same vacuum energy results. Choosing $r=h$ we obtain $b_{k}=h a_{h k^{\prime}}$ for all $k^{\prime} \mid n^{\prime}, n^{\prime}=n / h$ and vacuum energy $E_{0}^{\prime}=\mp 1 / n^{\prime}$. This agrees with the discussion on the modified torus construction in Sect. 4 , i.e. the sector twisted by $g^{h}$ of order $n^{\prime}$ is without a global phase anomaly. It is therefore necessary to only consider $\bar{a}$ with $h=1$ from now on.

Suppose next that $h=1$ and $\bar{b}=\bar{a}^{r}$ with $\left(n^{\prime}, r\right)=1$, i.e. $n^{\prime}=e$, where $e \| n$ and $r=c f$ with $f=n / e,(c, e)=1$. Then we find that $(k, r)=k_{f}$ and $b_{k^{\prime}}= \pm b_{e / k^{\prime}}$ according to (5.30) or (5.31). Thus the $\bar{b}$ sector vacuum energy is $\mp 1 / e$ in agreement with the Vacuum Conjectures. Likewise, one can show that for $\left(n^{\prime}, r\right) \neq 1,(5.30)$ can never be satisfied and therefore, the $\bar{b}$ sector cannot be tachyonic. The correct tachyonic 
singularity is therefore provided by (5.30) in each case. The residue of this singularity giving the vacuum degeneracy $n_{a}^{1 / 2}$ is discussed below.

We can also demonstrate the closure property of the Vacuum Conjectures from the symmetry relation (5.30). Thus if (5.30) is obeyed for $e_{1} \| n$ and $e_{2} \| n$ then $a_{k}$ obeys (5.30) for $e_{3}=e_{1} e_{2} /\left(e_{1}, e_{2}\right)^{2}$. This can be checked directly by applying (5.30) for $e_{1}$ followed by $e_{2}$ and showing that the resulting symmetry relation is that for $e_{3}$. Alternatively, $W_{e_{3}}=W_{e_{1}} W_{e_{2}}$ preserves $\eta_{\bar{a}}$ covariantly and so (5.30) is obeyed for $e_{3}$.

Let us turn now to the vacuum degeneracy $n_{\bar{a}}^{1 / 2}=D^{1 / 2} / V_{\bar{a}}$. For a tachyonic twisted sector with $h=1$ we expect (5.30) to be obeyed for $e=n$ to obtain (5.32). Substituting into (5.15) we obtain $D=n^{d / 2}$ in these cases. Therefore, the tachyon is unique $\left(n_{\bar{a}}=1\right)$ provided the invariant lattice volume obeys

$$
V_{\bar{a}}=n^{d / 4} \text {. }
$$

If $d=0$ then this is trivially true and $T_{a}^{(+)}=1 / \eta_{a}$ is $W_{n}$ invariant (but is inverted by some $W_{e}$ ). For $d \neq 0,(5.34)$ is a non-trivial condition on $\Lambda_{\bar{a}}$. The pole residues are now correctly specified by (5.34) and therefore, $T_{a}^{(+)}$is a hauptmodul for an appropriate fixing group $\Gamma_{0}(n)+e_{1}, e_{2}, \ldots$. If $T_{\bar{a}}^{(+)}$is invariant under all $\mathrm{AL}$ involutions then (5.30) is obeyed for all $e \| n, h=1$ and $\Theta_{\bar{a}} \neq 1$. In particular, $W_{n}$ invariance implies that $\Theta_{\bar{a}}^{*}(n \tau)=\Theta_{\bar{a}}(\tau)$. Therefore, $\Lambda_{\bar{a}}$ must be $\sqrt{n}$ self-dual, i.e. $\Lambda_{\bar{a}} \equiv \sqrt{n} \Lambda_{\bar{a}}^{*}$ for which (5.34) is naturally satisfied. This is a non-trivial property of such invariant sublattices of the Leech lattice.

Finally, let us consider as an example, the form of $T_{\bar{a}}^{(+)}$for $\bar{a}$ of prime order $p$. All prime divisors of the Conway group $\cdot 0$ satisfy $(p \pm 1) \mid 24$ [23] which corresponds to $W_{p}$ invariance (inversion) as follows. Suppose that $d=0$ and so $\bar{a}$ has no unit eigenvalues. Then the characteristic polynomial (5.8) is completely determined with $a_{p}=-a_{1}=r$, where $(p-1) r=24$. Therefore, (5.31) is obeyed for $h=1, e=p$ giving $T_{\bar{a}}^{(+)}=(\eta(\tau) / \eta(p \tau))^{r}$ which is inverted by $W_{p}$ and is the hauptmodul for $\Gamma_{0}(p)$ as stated in (3.14).

If $d \neq 0$ then applying (5.30) for $e=p$ gives $a_{p}=a_{1}=s$ with $(p+1) s=24$. Then $T_{\bar{a}}^{(+)}=\Theta_{\bar{a}} /(\eta(\tau) \eta(p \tau))^{s}$ is a hauptmodul for $\Gamma_{0}(p)+$, where $\Lambda_{\bar{a}}$ is a $2 s$-dimensional $\sqrt{p}$ self-dual lattice. For $p=2$ we have $\Theta_{\bar{a}}=\left(\theta_{3} \theta_{4}\right)^{8}+2^{-4} \theta_{2}^{16}[18]$ whereas for $p>2$ a more suitable closed form can be computed from the $\Gamma_{0}(p)$ invariant sum of the three remaining traces in (5.14). Then we find

$$
\psi(\tau)+\frac{2^{s}}{\psi(\tau / 2)}-\frac{2^{s} \psi(\tau / 2)}{\psi(\tau)}
$$

with $\psi(\tau)=(\eta(\tau) \eta(p \tau)) / \eta(2 \tau) \eta(2 p \tau))^{s}$ and where $\operatorname{Tr}\left( \pm a_{T}\right)= \pm 2^{s}$ is found by $\Gamma_{0}(p)$ invariance. It is easy to check that (5.35) is $\Gamma_{0}(p)+$ invariant for $(p+1) s=24$ with a unique pole at $q=0$ and is therefore a hauptmodul for $\Gamma_{0}(p)+$.

\section{General Remarks and Conclusions}

We have discussed the relationship between the Moonshine properties and an orbifold construction based on Monster group elements. We have shown that the correct vacuum structure for the corresponding twisted sectors guarantees the hauptmodul property for any Thompson series and the Power Law Formula observed by Conway and Norton. We will now briefly discuss a number of other features of this orbifold construction that we will expand on elsewhere [28]. 
In [8] it was noted that the remarks of Norton in the appendix of [20] about Generalised Moonshine also fit into an orbifold picture very naturally. Generalised Moonshine refers to the hauptmodul properties of a generalised Thompson series $T\left(g_{1}, g_{2}\right)$ given by the orbifold contribution

$$
T\left(g_{1}, g_{2}\right)(\tau)=g_{1} \square
$$

where $g_{1}, g_{2}$ are two elements of $M$ that commute. Then, as stated in [20], $T\left(g_{1}, g_{2}\right)$ is unchanged under simultaneous conjugation of $g_{1}$ and $g_{2}$. In addition, $T\left(g_{1}, g_{2}\right)$ transforms in the expected way under a modular transformation $\tau \rightarrow(a \tau+b) /(c \tau+d)$ with $T\left(g_{1}, g_{2}\right) \rightarrow T\left(g_{1}^{d} g_{2}^{-b}, g_{1}^{-c} g_{2}^{a}\right)$. We can also give the modular invariance group for $T\left(g_{1}, g_{2}\right)$ as follows. Let $n_{1}\left(n_{2}\right)$ be the order of $g_{1}\left(g_{2}\right)$ and let $h_{1}\left(h_{2}\right)$ be the corresponding divisor that describes the global phase anomaly for the sector twisted by $g_{1}\left(g_{2}\right)$. Then $T\left(g_{1}, g_{2}\right)$ is $T^{n_{2}}$ invariant up to a phase $e^{ \pm 2 \pi i / h_{2}}$ and $S T^{n_{1}} S$ invariant up to a phase $e^{ \pm 2 \pi i / h_{1}}$. These phases are determined by the vacuum energy for the sector twisted by $g_{2}$ and $g_{1}$, respectively. In general, we expect $T\left(g_{1}, g_{2}\right)$ to be invariant up to phases under

$$
\Gamma\left(n_{2}, n_{1}\right)==\left\{\left(\begin{array}{ll}
a & b \\
c & d
\end{array}\right)=\left(\begin{array}{ll}
1 \bmod n_{2} & 0 \bmod n_{2} \\
0 \bmod n_{1} & 1 \bmod n_{1}
\end{array}\right)\right\} .
$$

Finally, any singular behaviour of $T\left(g_{1}, g_{2}\right)$ at a rational cusp point $a / c$ is again determined by the presence of a tachyon in the sector twisted by $g_{1}^{-c} g_{2}^{a}$. The hauptmodul property for some appropriate group is then expected to follow from a similar argument as before.

In all our considerations thus far we have not examined the nature of the Monster partition function but have only concentrated on particular trace contributions to the full theory. Let us consider the example of the orbifold found by quotienting with respect to a $Z_{p}$ subgroup of $M$ generated by an element $g$ of prime order $p$. The full modular invariant partition function $Z_{\text {orb }}(\tau)$ for this theory is given by $[6,7]$

$$
Z_{\text {orb }}(\tau)=\frac{1}{p} \sum_{l, k=0}^{p-1} g^{k} \square_{g^{l}}
$$

With $p$ prime we can determine all of these traces from the Thompson series for $g$ since $T_{g}(\tau)=T_{g^{l}}(\tau)$ for $l=1,2, \ldots, p-1$ which implies that $g^{k} \square_{g^{l}}=T^{k} S\left(T_{g}(\tau)\right)$ and so

$$
Z_{\text {orb }}(\tau)=\frac{1}{p} J(\tau)+\frac{p-1}{p}\left(1+\sum_{k=0}^{p-1} T^{k} S\right) T_{g}(\tau)
$$

where $J(\tau)$ is the modular invariant partition function $1 \square$ for the Monster Module $V^{\natural}$. The remaining terms sum to form a modular invariant function with leading coefficient $(p-1) / p$ for the $q^{-1}$ pole and therefore the sum gives $\frac{p-1}{p} J(\tau)+C$ for some constant $C$ according to the Hauptmodul Theorem again. $p$

Suppose that the Thompson series $T_{g}$ is inverted by the Fricke involution $W_{p}$. Then the $g$ twisted space has no tachyon and $T_{g}$ is given by (3.14), where $(p-1) \mid 24$ and $g$ corresponds to a Leech lattice automorphism without any fixed points. We then find that the partition function (6.3) becomes $Z_{\text {orb }}(\tau)=J(\tau)+24$, where the constant is given by $C=(p-1) r=24$. Thus the partition function coincides with that of the original theory defined on $T^{24}=R^{24} / \Lambda$. On the other hand, if the 
Thompson series is invariant under $W_{p}$ (so that the $g$ twisted space contains a unique tachyon of energy $-1 / p)$ then $(6.3)$ becomes $Z_{\text {orb }}(\tau)=J(\tau)$, i.e. the partition function is unchanged by this orbifolding procedure.

FLM have conjectured that the Monster Module is the unique (bosonic) CFT with light-cone central charge 24 (giving the tachyon of mass -1) without massless states [1]. This characterisation is analogous to the unique determination of the Leech lattice as the only even self-dual lattice in 24 dimensions without any norm 2 vectors. On the basis of the prime ordered examples above and this conjecture we therefore make the following three suggestions (in an increasing order of speculation):

(a) The Monster Module can be alternatively constructed from the original untwisted theory on $T^{24}=R^{24} / \Lambda$ by $Z_{p}$ orbifolding with respect to prime ordered Leech lattice automorphisms without any fixed points. The resulting partition function is easily computed from the untwisted Thompson series $T_{g}^{(+)}$as above to give $J(\tau)$. A further $Z_{p}$ orbifolding of this theory then returns us to the untwisted theory on $T^{24}$ again explaining our result above.

(b) Orbifolding the Monster Module either returns the same Monster Module theory or brings us back to the original untwisted theory again (at least for prime ordered automorphisms). Whether this property can be extended to all orbifoldings of the Monster Module is now under investigation.

(c) Given that (b) is true for all orbifoldings, we are led to conjecture that the Moonshine properties are in fact due to the uniqueness of the Monster Module, i.e. the constraints placed on a Thompson series by (b) are sufficient to ensure the Vacuum Conjectures and hence the Moonshine properties follow. This would certainly offer the most satisfactory explanation for Monstrous Moonshine.

Apart from these more general considerations, the most immediate unanswered question is how do we construct the twisted sector associated with a general Monster group element in order to verify the Vacuum Conjectures? The most promising starting point is surely a vertex operator approach beginning with a centrally extended lattice automorphism as discussed in Sect. 5 (which does not appear to be known) before considering a general Monster element. The theory of generalised Kac-Moody algebras can be expected to play a central role here [5].

In conclusion, we would like to remark that, according to the uniqueness conjecture of FLM above, the Monster Module is the unique string theory without any massless states and therefore possesses no gauge or gravitational symmetry. We can speculate that this theory describes the state of the early universe before some phase transition sets in producing the necessary gauge bosons, graviton, etc. Leaving such a possibility aside, it is somewhat ironic that the string theory with the least degree of physical content has such an interesting mathematical one.

\section{Appendix. The Parabolic Cusps Points of $\mathscr{F}_{N}$}

In this appendix we will discuss the inequivalent parabolic cusps of $\mathscr{F}_{N}=H / \Gamma_{0}(N)$. In general, every rational point is a parabolic cusp point of some copy of $\mathscr{F}_{N}$ in $H$. The following lemma describes a convenient set of inequivalent cusps [29].

Lemma. Let $r / S$ be a rational number with $(r, S)=1$. For a given $N$ define

$$
k=(N, S), \quad N=k n, \quad S=k s .
$$


Then under $\Gamma_{0}(N)$ we have

$$
\begin{aligned}
\frac{r}{k s} & \sim \infty, & & k=N, \\
& \sim 0, & & k=1, \\
& \sim \frac{l}{k}, & & k \neq 1, N,
\end{aligned}
$$

where $0<l<k,(l, k)=1$ and $l$ is a solution of

$$
l \bmod m=r s \bmod m, \quad m=(n, k) .
$$

The proof of this lemma is as follows. If $k=N$ then $(r, N s)=1$ implies that for some $b, d \in Z$ we have $r d-N s b=1$. Therefore, $\gamma=\left(\begin{array}{cc}r & b \\ N s & d\end{array}\right) \in \Gamma_{0}(N)$ and $\gamma(\infty)$ $=r / N s$ as in (A.2a).

If $k \neq 1$ then for $\gamma=\left(\begin{array}{cc}a & b \\ N c & d\end{array}\right) \in \Gamma_{0}(N)$ we have $\gamma(r / k s)=r^{\prime} / k s^{\prime}$ for $r^{\prime}=a r+b k s$ and $s^{\prime}=n c r+d s$ with $\left(r^{\prime}, s^{\prime} k\right)=1$. It follows that

$$
r^{\prime} s^{\prime} \bmod m=r s \bmod m
$$

for $m=(n, k)$. We will now construct $\gamma$ such that $s^{\prime}=1$ and $r^{\prime}=l$ with $0 \leqq l<k$, i.e. $a, b, c, d$ obey

$$
\begin{gathered}
a=s+n l c, \\
k b=-r+l d .
\end{gathered}
$$

If $k=1$ then (A.5) is satisfied for $l=0$ giving (A.2b). If $k \neq 1$ then we will show that (A.5b) can always be solved. We begin by noting that $(n r, s)=1$ which implies that there exists $c, d$ such that $c n r+d s=1$. If $c_{0}, d_{0}$ are solutions of this then the general solution is $c=c_{0}+j s, d=d_{0}-j n r$ for any integer $j$. We will show that $d$ satisfies

$$
l d \bmod k=r \bmod k
$$

for some $0<l<k$ so that (A.5b) can be solved for $b$.

Let $k=p q$ with $(n, p)=1$ and $(n, q) \neq 1$, where $p$ is maximal in the sense that all prime divisors of $q$ divide $n$. Since $(r, k)=1$ and $(n, p)=1$ we have that $(n r, p)=1$. Therefore, $j(n r) \bmod p$ takes on all possible values for $0 \leqq j<p-1$. In particular, choose $j$ such that $j n r \bmod p=\left(d_{0}-1\right) \bmod p$, i.e. $d=1 \bmod p$ for $d=d_{0}-j n r$ which implies that $(d, p)=1$.

We also know that $(d, n)=1$ implying that $(d, q)=1$. Thus $(d, k)=1$ for the choice of $d$ above. Therefore, $l d \bmod k$ takes on all possible values in $\{1, \ldots, k-1\}$ for $0<l<k$. In particular, choose $l$ such that (A.6) is satisfied implying that $(l, k)=1$. Hence we can solve (A.5b) for $b$ giving $\gamma \in \Gamma_{0}(N)$ with $\gamma(r / k s)=l / k$ with $l$ as in (A.3). This concludes the proof.

The inequivalent cusps of $\mathscr{F}_{N}$ are therefore determined by all $k \mid N$ and all $0<l<k$ satisfying (A.3). We will illustrate this with a number of examples.

$N$ prime: If $N$ is prime then $k=1$ or $N$ giving only two inequivalent cusps $\infty$ and 0 of (A.2a) and (A.2b) as is well known.

AL cusps: If $e \| N$ [i.e. $(e, f)=1$ for $f=N / e$ ] then for $k=f$ we have $m=1$ and (A.3) implies that $r / f s \sim l / f \sim 1 / f$ for all $0<l<k$. Such a cusp point is defined in Sect. 4 as the Atkin-Lehner cusp for the AL transformation $W_{e}$. 
As an example of a general situation we can calculate the inequivalent cusps for $N=36$. The divisors of $N$ are $k=(1,2,3,4,6,9,12,18,36)$ with corresponding values of $m=(1,2,3,1,6,1,3,2,1)$. There are 12 inequivalent cusps determined by (A.2) and (A.3) as follows

$$
0, \frac{1}{2},\left\{\frac{1}{3}, \frac{2}{3}\right\}, \frac{1}{4},\left\{\frac{1}{6}, \frac{5}{6}\right\}, \frac{1}{9},\left\{\frac{1}{12}, \frac{5}{12}\right\}, \frac{1}{18}, \infty
$$

respectively where $0, \frac{1}{4}$, and $\frac{1}{9}$ are the AL cusps for $e=1,9,4$. Likewise, the action of the elements of $\mathscr{N}(36)$ on the cusps can be computed. Thus the Fricke involution $W_{36}: \tau \rightarrow-1 / 36 \tau$ acts as follows: $\left(0, \frac{1}{2}, \frac{1}{3}, \frac{2}{3}, \frac{1}{4}, \frac{1}{6}\right) \rightarrow\left(\infty, \frac{1}{18}, \frac{5}{12}, \frac{1}{12}, \frac{1}{9}, \frac{5}{6}\right)$ where in particular $\frac{1}{3} \rightarrow-\frac{1}{12} \sim \frac{5}{12}(m=3,-1=5 \bmod 3), \frac{2}{3} \rightarrow-\frac{1}{2.12} \sim \frac{1}{12}(m=3,-1.2=1 \bmod 3)$ and $\frac{1}{6} \rightarrow-\frac{1}{6} \sim \frac{5}{6}(m=6,-1=5 \bmod 6)$. Therefore, $\Gamma_{0}(36)+36$ has 6 cusps.

Acknowledgements. The author would like to thank Janos Balog and Noel Gorman for a number of useful discussions and Richard Borcherds for his helpful comments.

\section{References}

1. Frenkel, I., Lepowsky, J., Meurman, A.: A moonshine module for the monster. In: Lepowsky, J. et al. (eds.). Vertex operators in mathematics and physics, Berlin, Heidelberg, New York: Springer 1985; Vertex operator algebras and the Monster. New York: Academic Press 1988

2. Griess, R.: The friendly giant. Invent. Math. 69, 1 (1982)

3. Conway, J.H., Norton, S.P.: Monstrous moonshine. Bull. Lond. Math. Soc. 11, 308 (1979)

4. Borcherds, R.E.: University of Cambridge DPMMS preprint, 1989

5. Borcherds, R.E.: Generalized Kac-Moody algebras. J. Algebra 115, 501 (1988)

6. Dixon, L., Harvey, J.A., Vafa, C., Witten, E.: Strings on orbifolds. Nucl. Phys. B 261, 678 (1985)

7. Dixon, L., Harvey, J.A., Vafa, C., Witten, E.: Strings on orbifolds II. Nucl. Phys. B 274, 285 (1986)

8. Dixon, L., Ginsparg, P., Harvey, J.: Beauty and the beast: Superconformal symmetry in a Monster Module. Commun. Math. Phys. 119, 285 (1988)

9. Narain, K.S.: New heterotic string theories in uncompactified dimensions <10. Phys. Lett. 169 B, 41 (1986)

10. Conway, J.H., Sloane, N.J.A.: Sphere packings, lattices and groups. Berlin, Heidelberg, New York: Springer 1988

11. Goddard, P., Olive, D.: Algebras, lattices and strings. In: Lepowsky, J. et al. (eds.). Vertex operators in mathematics and physics. Berlin, Heidelberg, New York: Springer 1985

12. Serre, J-P.: A course in arithmetic. Berlin, Heidelberg, New York: Springer 1970

13. Goddard, P.: Meromorphic conformal field theory. In: Proceedings of the CIRM-Luminy Conference, 1988. Singapore: World Scientific 1989

14. Vafa, C.: Modular invariance and discrete torsion on orbifolds. Nucl. Phys. B 273, 592 (1986)

15. Narain, K.S., Sarmadi, M.H., Vafa, C.: Asymmetric orbifolds. Nucl. Phys. B 288, 551 (1987)

16. Thompson, J.G.: Finite groups and modular functions. Bull. Lond. Math. Soc. 11, 347 (1979)

17. Gunning, R.C.: Lectures on modular forms. Princeton, NJ: Princeton Univ. Press 1962

18. Moore, G.: Atkin-Lehner symmetry. Nucl. Phys. B 293, 139 (1987); Erratum. Nucl. Phys. B 299, 847 (1988)

19. Balog, J., Tuite, M.P.: The failure of Atkin-Lehner symmetry for lattice compactified strings. Nucl. Phys. B 319, 387 (1989)

20. Mason, G. (with an appendix by Norton, S.P.): Finite groups and modular functions. Proc. Symp. Pure Math. 47, 181 (1987) 
21. Apostol, T.M.: Modular functions and Dirichlet series in number theory. Berlin, Heidelberg, New York: Springer 1976

22. Ogg, A.P.: Hyperelliptic modular curves. Bull. Soc. Math. France 102, 449 (1974)

23. Conway, J.H., Curtis, R.T., Norton, S.P., Parker, R.A., Wilson, R.A.: An atlas of finite groups. Oxford: Clarendon Press 1985

24. Tits, J.: Normalisateurs de tores I. Groups de Coxeter étendus. J. Algebra 4, 16 (1966)

25. Dolan, L., Goddard, P., Montague, P.: Conformal field theory, triality and the Monster group. Preprint RU89/b1/45, DAMTP 89-29 (1989)

26. Sorba, P., Torresani, B.: Twisted vertex operators and string theory. Int. J. Mod. Phys. 3, 1451 (1988)

27. Corrigan, E., Holowood, T.H.: Comments on the algebra of straight, twisted and intertwining vertex operators. Nucl. Phys. B 304, 77 (1988)

28. Tuite, M.P.: To appear. DIAS preprint, 1990

29. Shimura, G.: Introduction to the arithmetic theory of automorphic functions. Princeton, NJ: Princeton Univ. Press 1971

Communicated by N. Yu. Reshetikhin 
